\title{
Nanotubular Titanium Oxynitride with an Ultra-Low Iridium Loading as a High-Performance Oxygen-Evolution-Reaction Thin-Film Electrode
}

Marjan Bele, ${ }^{1}$ Primož Jovanovič, ${ }^{1, *}$ Živa Marinko, ${ }^{2,3}$ Sandra Drev, ${ }^{4}$ Vid Simon Šelih, ${ }^{5}$ Janez Kovač, ${ }^{6}$ Miran Gaberšček, ${ }^{1, *}$ Gorazd Koderman Podboršek, ${ }^{1,3}$ Goran Dražić, ${ }^{1}$ Nejc Hodnik, ${ }^{1,3}$ Anton Kokalj, ${ }^{7,3}$ Luka Suhadolnik ${ }^{2, *}$

${ }^{1}$ Department of Materials Chemistry, National Institute of Chemistry, Hajdrihova 19, SI-1000 Ljubljana, Slovenia

${ }^{2}$ Department for Nanostructured Materials, Jožef Stefan Institute, Jamova 39, SI-1000 Ljubljana, Slovenia

${ }^{3}$ Jožef Stefan International Postgraduate School, Jamova 39, SI-1000 Ljubljana, Slovenia

${ }^{4}$ Center for Electron Microscopy and Microanalysis, Jožef Stefan Institute, Jamova 39, SI-1000 Ljubljana, Slovenia

${ }^{5}$ Department of Analytical Chemistry, National Institute of Chemistry, Hajdrihova 19, SI-1000 Ljubljana, Slovenia

${ }^{6}$ Department of Surface Engineering and Optoelectronics, Jožef Stefan Institute, Jamova 39, SI-1000 Ljubljana, Slovenia

${ }^{7}$ Department of Physical and Organic Chemistry, Jožef Stefan Institute, Jamova 39, SI-1000 Ljubljana, Slovenia

* Corresponding authors: Dr. Luka Suhadolnik, e-mail: luka.suhadolnik@ijs.si Prof. Miran Gaberšček, e-mail: miran.gaberscek@ki.si Dr. Primož Jovanovič, e-mail: primoz.jovanovic@ki.si

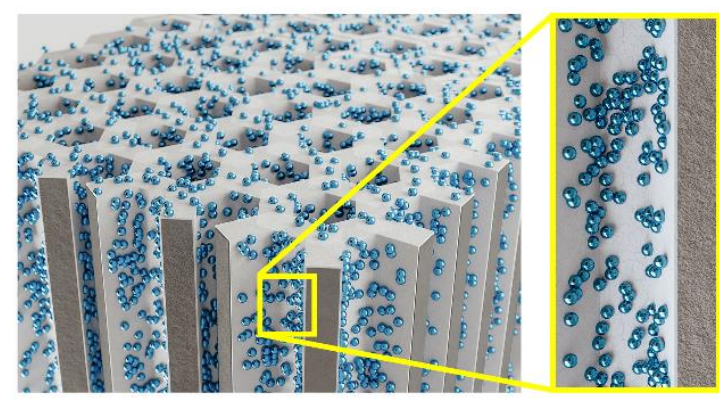

\section{Abstract}

The present study targets one of the grand challenges of electrochemical hydrogen production: a durable and cost-effective oxygen-evolution catalyst. We present a thin-film composite electrode with a unique morphology and an ultra-low loading of iridium that has extraordinary 
electrocatalytic properties. This is accomplished by the electrochemical growth of a defined, high-surface-area titanium oxide nanotubular film followed by the nitridation and effective immobilization of iridium nanoparticles. The applicative relevance of this production process is justified by a remarkable oxygen-evolution reaction (OER) activity and high stability. Due to the confinement inside the pores and the strong metal-support interaction (SMSI) effects, the OER exhibited a higher turnover. The high durability is achieved by self-passivation of the titanium oxynitride (TiON) surface layer with $\mathrm{TiO}_{2}$, which in addition also effectively embeds the Ir nanoparticles, while still keeping them electrically wired. An additional contribution to the enhanced durability comes from the nitrogen atoms, which according to our DFT calculations reduce the tendency of the Ir nanoparticles to grow. We also introduce an advanced electrochemical characterization platform for the in-depth study of thin-film electrodes. Namely, the entire process of the TiON-Ir electrode's preparation and the electrochemical evaluation can be tracked with scanning electron microscopy, X-ray diffraction (XRD) and Xray photoelectron spectroscopy (XPS) at identical locations. In general, the novel experimental approach allows for the unique morphological, structural and compositional insights into the preparation and electrocatalytic performance of thin films, making it useful also outside electrocatalysis applications.

\section{Keywords}

Electrocatalysis; Oxygen evolution reaction; TiON-Ir-nanotube catalyst; Thin-film electrode; Titanium oxynitride nanotubular support; TiON-Ir catalyst; Iridium nanoparticles; IL-SEM

\section{Broader context}

Electrochemical conversion of renewable resources to electrical energy, fuels or useful chemicals is one of the most promising directions for humankind to meet the most urgent technological goals, namely, a clean energy landscape. Electrochemical water splitting is considered as a cornerstone of such scenario delivering hydrogen as a clean energy carrier for future power supply, energy storage matrix and raw commodity for chemical sector. Further expansion of proton exchange membrane electrolysers (PEM) as the most promising platform for sustainable hydrogen generation, is jeopardized by its dependency on noble metal catalysts. Presently, the crucial bottleneck is the anode side of electrolyser where the sluggish oxygen evolution reaction (OER) dictates employment of expensive and scarce iridium in unsustainable amount. There is therefore a strong incentive to minimize the amount of iridium and at the same time enhance its activity and durability. We demonstrate that both aspects are well addressed 
by employing nanostructured iridium moieties immobilized on a titanium oxy-nitride substrate. Such a combination offers an elegant platform for tailoring the electrode architecture. We showcase that confinement inside the pores and strong metal-support interaction retain iridium utilization during electrochemical operation.

\section{Introduction}

In the field of electrocatalysis, catalytic materials are utilized in the form of a high-surface-area, highly conductive thin film. The benefits of such materials are the good accessibility of the reactants and the assurance of good electrical contacts with the supporting material. However, the design of such materials is often challenging due to the harsh electrochemical environment that leads to degradation of the thin-film catalyst. ${ }^{1}$ This is especially true for the oxygenevolution reaction (OER), where electrochemical conditions are more severe in comparison to other reactions of the energy-conversion sector. Iridium is the material of choice for OER. ${ }^{2,3}$ But due to its high price, it must be dispersed on a substrate with a high surface area to increase the utilization of the catalyst layer. ${ }^{4-7}$ Among OER thin-film approaches, the state of the art are still dimensionally stable anodes (DSAs) ${ }^{8}$ that are synthesized by the co-precipitation of $\mathrm{RuO}_{2}$ and $\mathrm{IrO}_{2}$ oxides supported by a titanium substrate with a thickness of a few micrometers. ${ }^{9}$ This results in a high crystallinity as well as mechanical and electrochemical stability, both of which meet industrial demands for long-term operation. Although these electrodes are very stable, they require high noble-metal loadings. As iridium is one of the scarcest metals on Earth as well as being geologically unevenly distributed (it is predominantly concentrated in the Republic of South Africa) it is of the highest importance to utilize it as effectively as possible if the technology of electrochemical hydrogen production is to be scaled up. For example, one of the best PEM electrolyzers in terms of iridium-anode loading $\left(0.25 \mathrm{mg} \mathrm{cm}^{-2}\right)^{10}$ obtained so far, still means that approximately 100 tons of iridium would be needed to produce devices that generate hydrogen at a rate equivalent to $1 \mathrm{TW}$ of energy storage $\left(1 \mathrm{TW}_{\mathrm{H} 2}\right)$. Considering that the current annual global production of iridium is merely $<10$ tons, ${ }^{11}$ only a significant improvement in iridium utilization can justify the economic feasibility of PEM electrolyzers.

In this respect, dispersing the Ir catalysts on a high-surface-area support would provide the desired lowering of the noble-metal loading. However, as carbon-based supports, otherwise the most commonly used in electrocatalysis, are unstable under OER electrochemical conditions, ${ }^{12-}$

${ }^{14}$ alternative solutions are needed. ${ }^{15}$ In order for such composites to be industrially relevant, their preparation should be as scalable and straightforward as possible. In this respect, the electrolyser technology would benefit even more if such a synthesis provided an immobilized catalyst that can be directly used as an electrode rather than a catalyst in the form of a powder. 
Considering the above requirements, electrochemical anodization (oxidation) seems an appropriate platform. ${ }^{16}$ Furthermore, this method enables the growth of oxide nanostructures of different morphologies. ${ }^{17}$ The most well-known and studied are $\mathrm{TiO}_{2}$ nanotubes, ${ }^{18}$ which, however, cannot be used for electrochemical applications exhibiting high current densities, since titanium dioxide is a semiconductor and thus might not provide sufficient electrical conductivity.

There have been many attempts to increase the electrical conductivity of $\mathrm{TiO}_{2}$ nanotubes. ${ }^{19}$ One of the most important ways to change the properties of titanium dioxide is its conversion to other compounds that can have different properties, including electrical conductivity. Examples of these materials include barium titanate ${ }^{20}$ and strontium titanate, ${ }^{21}$ both of which retain their nanotubular structure after the transformation during a hydrothermal treatment. These materials are used primarily for sensor applications. ${ }^{22}$ On the other hand, conductive titanium oxynitride is also a very promising candidate for electrocatalytic applications, ${ }^{23,24}$ however, it is rarely employed for OER. Besides our recent studies, ${ }^{11,25}$ there have been no other reports dealing with Ti oxynitrides in conjunction with Ir.

In this investigation we focus on the development of a novel thin-film electrode material for the oxygen-evolution reaction that exhibits an extraordinarily high OER activity and good stability. The beneficial properties of our thin-film OER electrode are in our opinion due to the strong metal support interaction or SMSI effect, which we support by experimental data and also the density functional theory (DFT) calculations. In order to obtain an in-depth insight into the development of the material's features during its synthesis, as well as the impact on the final properties, we used a unique characterization approach. Namely, for the first the entire process of the TiON-Ir electrocatalyst preparation and electrochemical operation was tracked with the so-called identical-location scanning electron microscopy (IL-SEM), X-ray diffraction (XRD) and X-ray photoelectron spectroscopy (XPS) techniques.

\section{Experimental}

\subsection{Synthesis of the TiON-Ir catalyst}

The TiON-Ir catalyst was prepared in immobilized form following the procedure shown in Figure 1. In the first step, the TiAl6V4 (90 wt.\% Ti, $6 \mathrm{wt} . \%$ Al, $4 \mathrm{wt} . \% \mathrm{~V}$ ) alloy in the shape of an SEM pin stub was subjected to potentiostatic anodization in a two-electrode electrochemical cell using a stainless-steel counter electrode (Figure S1a). The titanium alloy was first cleaned with acetone and ethanol in an ultrasonic bath and then anodized in an electrolyte consisting of 0.3 wt.\% $\mathrm{NH}_{4} \mathrm{~F}$ (99.99\%, Sigma-Aldrich) and 2 vol.\% deionized water in ethylene glycol 
(99.5\%, Carlo Erba Reagents). The anodizing voltage was kept constant at $60 \mathrm{~V}$ and the anodizing time was 30 minutes. The procedure resulted in an amorphous $\mathrm{TiO}_{2}$ nanotube film, which was then washed with deionized water and ethanol and annealed at $450{ }^{\circ} \mathrm{C}$ for 1 hour. After the conversion of the amorphous $\mathrm{TiO}_{2}$ phase to the anatase phase, a second annealing was performed in an ammonia atmosphere at $700{ }^{\circ} \mathrm{C}$ for $10 \mathrm{~h}$ to convert the crystalline $\mathrm{TiO}_{2}$ nanotube arrays into the TiON substrate. The flow of pure ammonia gas was kept constant at $50 \mathrm{~cm}^{3} \mathrm{~min}^{-1}$ and a pressure of 1 atmosphere. In the last step, iridium nanoparticles were deposited on the TiON substrate. To deposit the iridium particles on the TiON substrate, the following procedure was developed. First, $15 \mathrm{mg}$ of iridium (III) bromide hydrate (SigmaAldrich, St. Louis, MO) were dissolved in $1.5 \mathrm{~mL}$ of water at $50^{\circ} \mathrm{C}$. Then the solution was dipcoated on the TiON substrate with a withdrawal speed of $1 \mathrm{~cm} \mathrm{~s}^{-1}$ and dried at $50{ }^{\circ} \mathrm{C}$. Afterwards, the sample was thermally treated in a $5 \% \mathrm{H}_{2} / \mathrm{Ar}$ mixture. The temperature was increased with a rate of $2{ }^{\circ} \mathrm{C} \min ^{-1}$ to $400{ }^{\circ} \mathrm{C}$ for $1 \mathrm{~h}$, with the subsequent cooling rate to room temperature being $3{ }^{\circ} \mathrm{C} \min ^{-1}$.

To compare the activity of the TiON-Ir catalyst with the $\mathrm{TiO}_{2}$ - Ir catalyst, another sample was prepared using the same procedure, but without the nitridation step. The TiAl6V4 alloy was chosen as the starting material since it is the most commonly used titanium alloy known from a wide range of applications. To observe the morphological differences between the anodized TiAl6V4 alloy and the pure titanium, a Grade-2 titanium foil was anodized under the same conditions.

\section{TiAl6V4}

PIN STUB

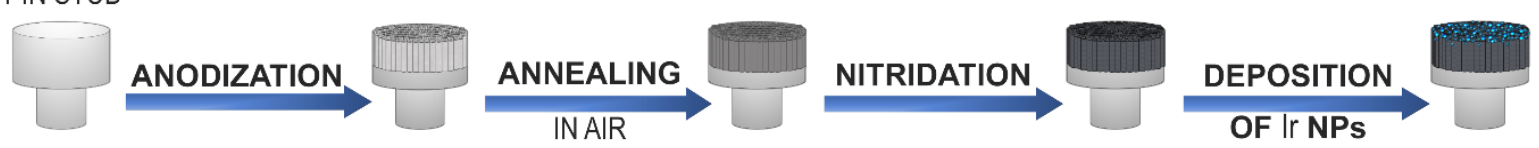

Figure 1: Procedure for preparing the immobilized TiON-Ir thin-film electrode.

\subsection{Materials characterization}

Each step of the synthesis process was studied with IL-SEM. ${ }^{26,27,28}$ In this way we were able to observe the same site on the sample throughout the entire synthesis process and after the electrochemical analysis. The evolution of the morphology was studied with a Zeiss FE-SEM SUPRA 35 VP field-emission scanning electron microscope (Carl Zeiss, Oberkochen, Germany). Detailed investigations of the $\mathrm{TiO}_{2}$ and $\mathrm{TiON}$ structural and elemental features were performed using a conventional 200-kV transmission electron microscope (JEM-2010F, Jeol, 
Japan). Chemical analyses were performed using a $\mathrm{Si}(\mathrm{Li})$ energy-dispersive spectroscopy (EDS) detector. Each step of the TiON-Ir preparation was also characterized by X-ray diffraction. The X-ray diffractograms of the samples were recorded on a flat, disc-like Si sample holder with a PANalytical X'Pert PRO MPD X-ray powder diffractometer (PANalytical B.V., Almelo, The Netherlands) with a radiation wavelength $\mathrm{Cu}-\mathrm{K}_{\alpha 1}=1.5406 \AA$ in the alphal configuration with a Johansson monochromator on the primary side. The diffractograms were recorded with a $0.034^{\circ}$ resolution and 100 -s signal-integration time in the $2 \theta$ range from $10^{\circ}$ to $80^{\circ}$ with a scanning $X^{\prime}$ Celerator detector (full open $2.122^{\circ} 2 \theta$ ). The 0.02 rad Soller slits and 10-mm divergent slits were used together with a 10-mm beam mask. The phases were identified using the X'Pert HighScore Plus program and the International Centre for Diffraction Data (ICDD) PDF-4+ 2016 database. ${ }^{29}$ The surface of the TiON-Ir catalyst (the upper 3-5 nm) was also characterized using X-ray photoelectron spectroscopy (XPS) on a PHI-TFA XPS spectrometer produced by Physical Electronics Inc and equipped with Al-monochromatic source. The sample was analyzed before and after the electrochemical measurements to observe the changes to the structure and the composition during the degradation protocol. BET surfacearea and nitrogen-sorption measurements of the $\mathrm{TiO}_{2}$ and $\mathrm{TiON}$ supports in powder form were recorded using a Tristar 3000 automated gas-adsorption analyzer (Micromeritics Instrument Corp.) at $77 \mathrm{~K}$. The BET surface area was calculated using the adsorption branch in the relative pressure range between 0.29 and 0 bar. The iridium content was determined according to the following protocol. The sample was submerged in $8 \mathrm{~mL}$ of hot aqua regia $\left(3: 1 \mathrm{HCl}: \mathrm{HNO}_{3} \mathrm{v} / \mathrm{v}\right.$, concentrated) and sonicated for 90 minutes. During this time, all the dark surface coating, containing elemental Ir and the underlying substrate, was etched away and removed into the aqua regia. The latter was subsequently diluted with milli-Q water to $50 \mathrm{~mL}$ and the Ir concentration was measured with an ICP-OES instrument (Varian 715-ES). The net mass of Ir on the sample was then calculated from the concentrations. In the case of the sample that was electrochemically analyzed, the mass of Ir was $0.85 \mu \mathrm{g}$, which resulted in an Ir loading of 1.7 $\mu \mathrm{g} \mathrm{cm}^{-2}$ geom. To confirm that the Ir was completely removed from the underlying substrate, we repeated the etching procedure. In this case the Ir signal was below the limit of detection.

\subsection{Electrochemical measurements}

IL-SEM electrodes: Experiments were performed in a single-compartment three-electrode cell with an $\mathrm{Ag} / \mathrm{AgCl}$ reference electrode and a graphite-rod counter electrode. The working electrode was positioned at the bottom of the cell with the catalyst surface facing upwards. The electrochemical cell used for the determination of catalytic activity was the same as the one used for the anodic oxidation of the starting TiAl6V4 pin stub in order to ensure there were no 
electrochemical contributions from the metal pin stub that held the electrocatalyst (Figure S1b). The working electrode consisted of a metallic holder with an SEM pin stub (the same as used in the synthesis of the catalytic composite). An aqueous solution of $0.1 \mathrm{M} \mathrm{HClO}_{4}$ (Merck, Suprapur, $70 \%$, diluted by Milli-Q, $18.2 \mathrm{M} \Omega \mathrm{cm}$ ) was used as the supporting electrolyte. In the electrochemical treatments, IR compensation was applied ( $85 \%$ was compensated for). All the potentials of the reported electrochemical experiments refer to the reversible hydrogen electrode (RHE). The RHE potential was measured by saturating the electrolyte with hydrogen and measuring the open-circuit potential (OCP). Prior to the electrochemical characterization of the TiON-Ir, the catalyst was pre-activated by a galvanostatic treatment $\left(5 \mathrm{~mA} \mathrm{~cm}^{-2}\right.$ geom for $30 \mathrm{~min}$ ). The activation step is necessary in order to diminish the Faradaic contribution of the TiON support under OER conditions (as shown in the continuation, the XPS analyses reveal that TiON undergoes a chemical modification due to electrochemical oxidation during the OER conditions). The OER activities of four Ir-based catalysts were evaluated using linear-sweep voltammetry (LSV) with a scan rate of $20 \mathrm{mV} \mathrm{s}^{-1}$ in the potential window between 0.3 and 1.8 $\mathrm{V}$. The degradation protocol consisted of a LSV potential increase from 0.3 to $1.8 \mathrm{~V}$ and holding the potential at $1.8 \mathrm{~V}$ for $60 \mathrm{~min}$. After the degradation protocol, LSV was performed under the same conditions as prior to the potentiostatic degradation. Such an electrochemical protocol was used to study the activity of all three prepared samples: two TiON-based Ir catalysts and a $\mathrm{TiO}_{2}$-based Ir catalyst. In the case of the TiON-Ir samples, one sample was used to study the morphological changes during each synthesis step and to observe the morphological changes after the electrochemical treatment. Another sample was prepared in order to compare its electrochemical performance with the $\mathrm{TiO}_{2}$-Ir analogue.

Electrochemical treatment of IL-SEM electrodes for XPS: XPS analyses were performed on a separate TiON-Ir sample, which was prepared in the same way as the primary TiON-Ir analogue. The XPS analyses were performed prior to and after the electrochemical protocol, which consisted of a 30 min potentiostatic (1.6 V vs RHE) treatment.

RDE measurements: The electrochemical performances were compared to a commercial Ir analogue, i.e., "Ir Black" (Alfa Aesar, 99.95 \% CAS: 7439-88-5). The electrochemical characterization of this sample was performed in a rotating-disc-electrode (RDE) configuration, as follows. A conventional three-electrode setup consisting of a gold disc electrode $(d=4 \mathrm{~mm})$, a graphite rod as the counter electrode and an $\mathrm{Ag} / \mathrm{AgCl}$ reference electrode was used. The last of these was separated from the main cell compartment by a Luggin capillary. De-aerated 0.1$\mathrm{M} \mathrm{HClO}_{4}$ (Merck, Suprapur, $70 \%$, diluted by Milli-Q, $18.2 \mathrm{M} \Omega \mathrm{cm}$ ) was used as the working electrolyte. For the preparation of the electrode, a standard ink containing $1.4 \mathrm{mg}$ of Ir was 
dispersed in $3 \mathrm{ml}$ of water, $0.96 \mathrm{ml}$ of 2-propanol, and $16 \mu \mathrm{l}$ of Nafion ( $5 \mathrm{wt} \%$, Sigma Aldrich). The ink was bath sonicated for $20 \mathrm{~min}$; following sonication, $20-\mu 1$ aliquots were pipetted onto polished polycrystalline gold electrodes. ${ }^{30}$ Before any activity measurements, the sample was electrochemically oxidized with 200 potential cycles $\left(300 \mathrm{mV} \mathrm{s}^{-1}\right)$ between 0.05 and $1.2 \mathrm{~V}$ vs RHE in order to provide for a stable CV. The electrolyte was changed before the activity measurement to avoid any trace of contamination. The OER activity was evaluated using the same protocol as in the case of the IL-SEM holder, i.e., LSV with a scan rate of $20 \mathrm{mV} \mathrm{s}^{-1}$ in a potential window between 0.3 and $1.8 \mathrm{~V}$. The degradation protocol consisted of a LSV potential increase from 0.3 to $1.8 \mathrm{~V}$ and holding the potential at $1.8 \mathrm{~V}$ for $30 \mathrm{~min}$. After the degradation protocol, LSV was performed under the same conditions as prior to the potentiostatic degradation. Throughout the entire electrochemical treatment, a rate of 2500 rotations per minute (RPM) was used. In the case of all the electrochemical treatments, IR compensation was applied (85\% was compensated for).

\subsection{DFT calculations}

DFT calculations were performed with the PWSCf code from the Quantum ESPRESSO distribution $^{31}$ using the GGA+U method ${ }^{32,33}$ with the exchange-correlation functional of Perdew-Burke-Ernzerhof (PBE). ${ }^{34}$ Core electrons were described with the projectoraugmented-wave (PAW) potentials, obtained from the pslibrary. ${ }^{35,36}$ Kohn-Sham orbitals were expanded in a plane-wave basis set with a kinetic energy cutoff of 50 Ry (575 Ry for the charge density). A $U$ parameter of $4.0 \mathrm{eV}$ was used for the Ti ions. This $U$ value was calculated self-consistently for the $\mathrm{Ti}_{1.5} \mathrm{ON}$ bulk using the $\mathrm{hp} . \mathrm{x}$ code that utilizes a density-functionalperturbation-theory scheme. ${ }^{37}$ The utilized model of $\mathrm{Ti}_{1.5} \mathrm{ON}$ bulk ${ }^{\ddagger}$ can be described with a rocksalt crystal structure with two interpenetrating fcc latices of $\mathrm{O} / \mathrm{N}$ anions and Ti cations (with $25 \%$ of vacancies); the two lattices are shifted by half a Bravais lattice vector with respect to one another. On average, there are 7 atoms ( $3 \mathrm{Ti}, 2 \mathrm{~N}, 2 \mathrm{O})$ and $1 \mathrm{Ti}$-vacancy in the unit cell (Figure S2a). Our calculated lattice parameter for the $\mathrm{Ti}_{1.5} \mathrm{ON}$ bulk is $4.172 \AA$, in good agreement with the experimental value. ${ }^{\ddagger}$ Brillouin-zone integrations were performed with the uniformly shifted $4 \times 4 \times 4 \mathrm{k}$-mesh for the $\mathrm{Ti}_{1.5} \mathrm{ON}$ bulk (for the surface calculations the $\mathrm{k}$-grids were of comparable quality) and a Methfessel-Paxton smearing ${ }^{38}$ of $0.02 \mathrm{Ry}$.

The HAADF-STEM experiments revealed that the dominant surface of the $\mathrm{Ti}_{1.5} \mathrm{ON}$ substrate is the (111). As a result, we described the $\mathrm{Ti}_{1.5} \mathrm{ON}$ substrate with the $\mathrm{Ti}_{1.5} \mathrm{ON}(111)$ slab. Its structure in the surface normal direction consists of Ti and $\mathrm{O} / \mathrm{N}$ layers stacked one on top of the other. The $\mathrm{Ti}_{1.5} \mathrm{ON}(111)$ was modeled with a symmetric non-polar slab consisting of five Ti- 
layers terminated by $\mathrm{O} / \mathrm{N}$ layers on both sides of the slab (Figure $\mathrm{S} 2 \mathrm{~b}$ ): to maintain the stoichiometry the surface $\mathrm{O} / \mathrm{N}$ layers contain only $50 \%$ of the $\mathrm{O}$ and $\mathrm{N}$ ions. The largest considered surface model consists of a $(3 \times 3)$ supercell of $\mathrm{Ti}_{1.5} \mathrm{ON}(111)$ with the supercell vectors $|\boldsymbol{a}|=|\boldsymbol{b}|=17.700 \AA$, which was described by the Gamma k-point. All degrees of freedom were relaxed during the calculations.

The bonding of the Ir clusters to the $\mathrm{Ti}_{1.5} \mathrm{ON}(111)$ was analyzed by means of the electroncharge-density difference, $\Delta \rho(\boldsymbol{r})$, calculated as:

$$
\Delta \rho(\boldsymbol{r})=\rho_{\mathrm{Ir}_{n} / \mathrm{TiON}}(\boldsymbol{r})-\rho_{\mathrm{Ir}_{n}}(\boldsymbol{r})-\rho_{\mathrm{TiON}}(\boldsymbol{r})
$$

where $\rho_{\mathrm{Ir}_{n} / \mathrm{TiON}}(\boldsymbol{r})$ is the electron charge density of the whole $\mathrm{Ir}_{n}$-cluster/Ti ${ }_{1.5} \mathrm{ON}(111)$ system, whereas $\rho_{\mathrm{Ir}_{n}}(\boldsymbol{r})$ and $\rho_{\mathrm{TiON}}(\boldsymbol{r})$ are the electron densities of the $\operatorname{Ir}_{n}$ cluster and the $\operatorname{Ti}_{1.5} \mathrm{ON}(111)$ slab, respectively, both having the same geometry as in the whole system.

The planar-integrated charge-density difference, $\Delta \rho(\mathrm{z})$, was calculated by integrating $\Delta \rho(\boldsymbol{r})$ by $x y$-slices spanned by the surface supercell, i.e.,

$$
\Delta \rho(z)=\int_{A} \Delta \rho(x, y, z) \mathrm{d} x \mathrm{~d} y
$$

where $A$ is the area spanned by the surface supercell and $\Delta \rho(x, y, z) \equiv \Delta \rho(\boldsymbol{r})$.

The adhesion energies of the $\mathrm{Ir}_{n}$ clusters on the $\mathrm{Ti}_{1.5} \mathrm{ON}(111)$ were calculated as:

$$
E_{\mathrm{adh}}=E_{\mathrm{Ir}_{n} / \mathrm{TiON}}-E_{\mathrm{Ir}_{n}}-E_{\mathrm{TiON}}
$$

where $E_{\mathrm{Ir}_{n} / \mathrm{TiON}}, E_{\mathrm{Ir}_{n}}$, and $E_{\mathrm{TiON}}$ are the total (potential) energies of the relaxed $\mathrm{Ir}_{n^{-}}$ cluster/ $\mathrm{Ti}_{1.5} \mathrm{ON}(111)$ system, the relaxed standalone $\mathrm{Ir}_{n}$ cluster, and the relaxed bare $\mathrm{Ti}_{1.5} \mathrm{ON}(111)$ slab, respectively.

For consistency reasons, with the designation used in the experimental counterpart of the article, the $\mathrm{Ti}_{1.5} \mathrm{ON}$ will be simply designated by the acronym $\mathrm{TiON}$ in the following. 


\section{Results and discussion}

\subsection{Characterization of the TiON-Ir nanostructures}

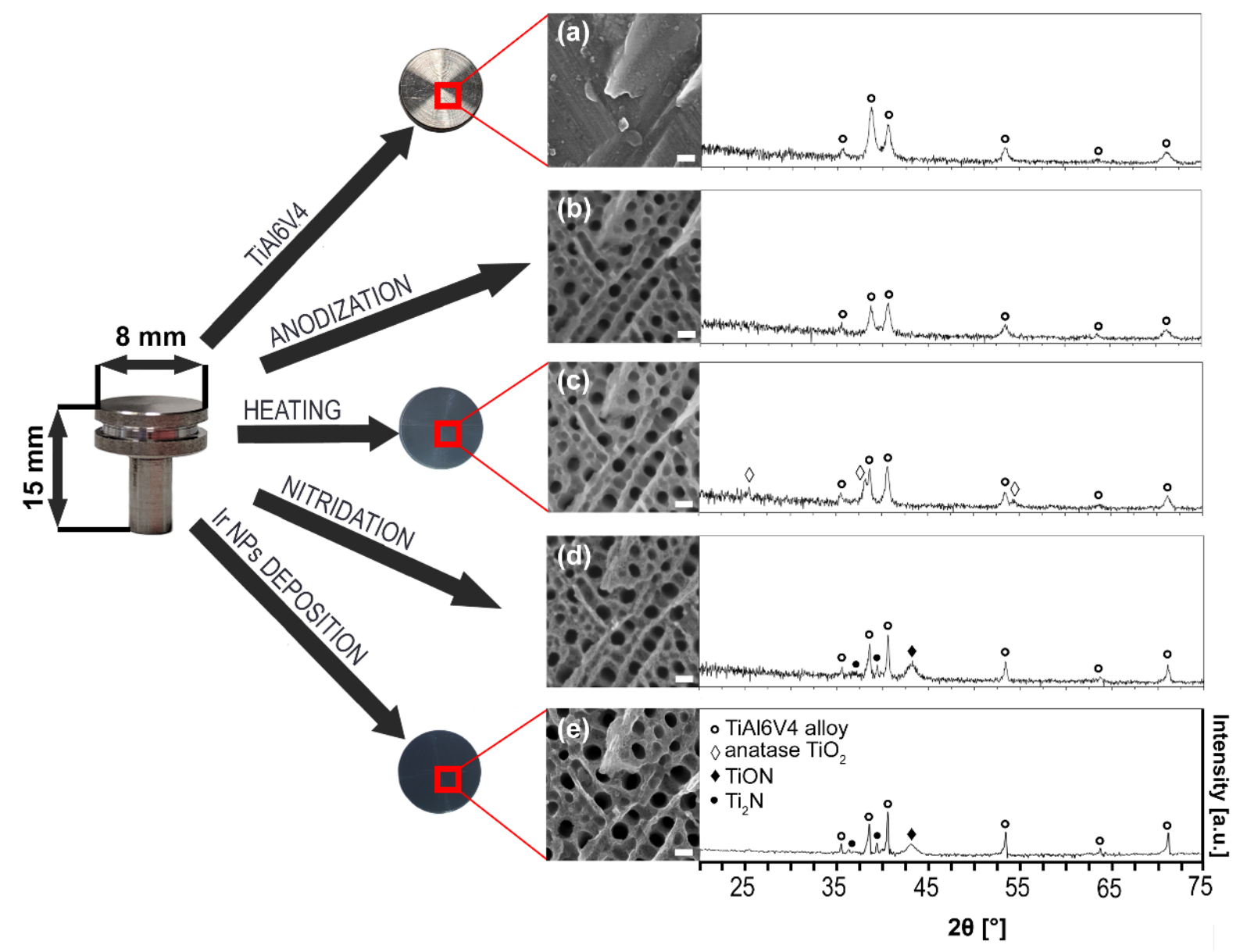

Figure 2: Top-view IL-SEM images (identical location) of our sample throughout the entire synthesis process and corresponding XRD analyses. (a) A starting TiAl6V4 alloy, (b) the anodized starting material, (c) $\mathrm{TiO}_{2}$ nanotubes annealed in air, (d) the sample after annealing in ammonia and (e) the final TiON-Ir catalyst. Scale $100 \mathrm{~nm}$.

Figure 2 shows the dimensions of the SEM pin stub together with the optical changes to its surface in three different phases of the TiON-Ir catalyst's preparation. The same figure also shows top-view SEM images of the same site of our sample throughout the entire synthesis process and the corresponding diffraction peaks from the XRD analyses. The surface color of the TiAl6V4 SEM pin stub is shown in Figure 2a. It was cleaned, but not polished, before use, which is why irregularities can be observed. After anodization, the surface changes to a highsurface-area, rigidly attached $\mathrm{TiO}_{2}$ nanotubular film (Figure $2 \mathrm{~b}$ ), which retains its morphology after annealing at $450{ }^{\circ} \mathrm{C}$ (Figure 2c). A particularly important characteristic of the sample is that the surface of the film does not crack during the calcination, as this allows for controlled thin-film preparation. Macroscopic cracks, however, always occur during the calcination of the anodized (pure) titanium (Figure 3b). In the case of the anodized TiAl6V4 alloy, they do not 
appear due to better matching of the temperature expansion coefficients of the substrate and the nanostructured film (Figure 3a). Both films shown in Figure 3 were annealed at $450{ }^{\circ} \mathrm{C}$ for $1 \mathrm{~h}$. Even in the next synthesis step, annealing in ammonia at $700{ }^{\circ} \mathrm{C}$, cracks do not occur. The second annealing further increases the specific surface area, as determined with the BET analysis of the $\mathrm{TiO}_{2}$ and $\mathrm{TiON}$ supports that were detached from the metal titanium substrate $\left(29.1 \pm 0.2 \mathrm{~m}^{2} \mathrm{~g}^{-1}\right.$ for $\mathrm{TiO}_{2}$ in comparison to $36.3 \pm 0.2 \mathrm{~m}^{2} \mathrm{~g}^{-1}$ for TiON). It also establishes a good electronic conductivity of the supporting TiON material (Figure $2 \mathrm{~d}$ ). ${ }^{39}$ In the last step, Ir nanoparticles were deposited; however, they are too small in terms of size and quantity to be visible in the SEM micrograph of Figure 2e. An animation, showing the morphological changes throughout the synthesis process, is available online in the Supplementary Data video clip.
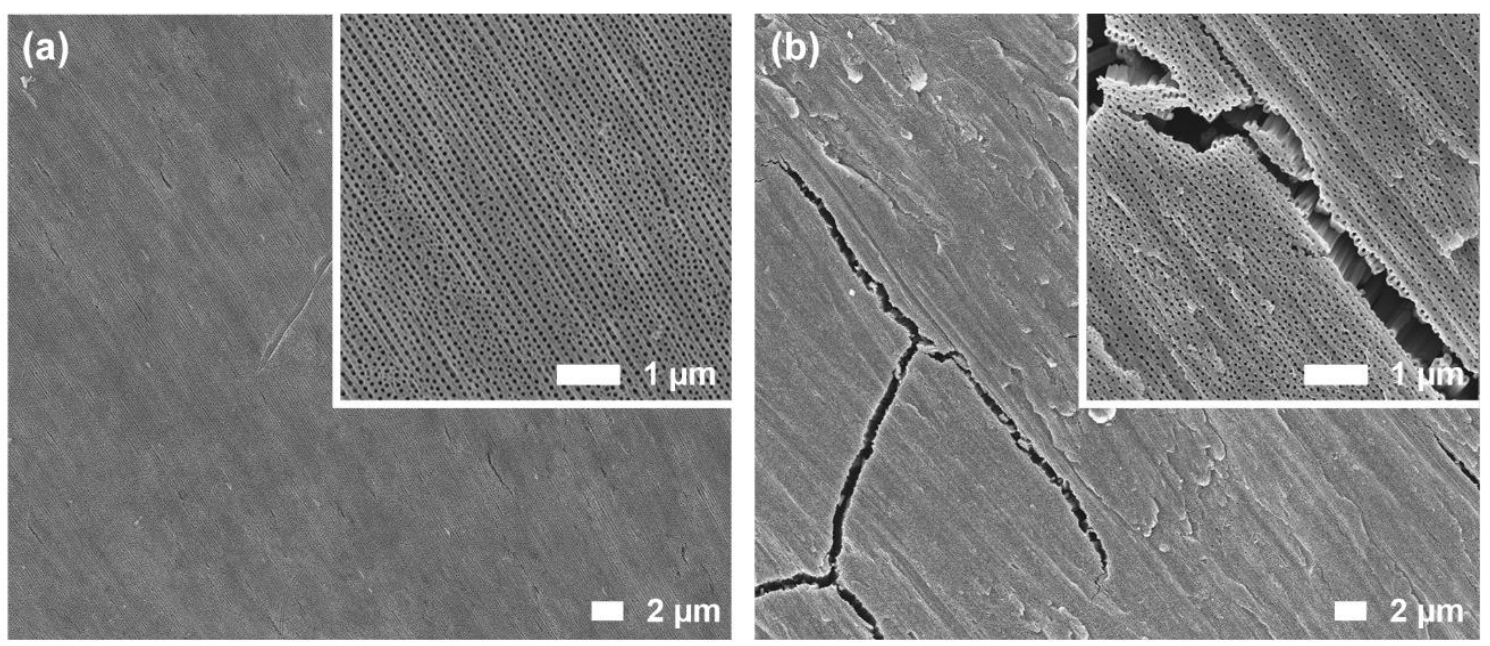

Figure 3. Comparison of $\mathrm{TiO}_{2}$ nanotube film grown on (a) TiAl6V4 alloy and (b) $\mathrm{TiO}_{2}$ nanotube film grown on titanium foil.

The inner and outer diameters of the $\mathrm{TiO}_{2}$ (Figure 4a) and $\mathrm{TiON}$ (Figure 5a) nanotubes estimated from the TEM top-sectional view of the nanotubes are approximately 70-80 $\mathrm{nm}$ and $100-120 \mathrm{~nm}$ in the case of the $\mathrm{TiO}_{2}$, and $60-80 \mathrm{~nm}$ and $110-120 \mathrm{~nm}$ in the case of the TiON. The thickness of the $\mathrm{TiO}_{2}$ nanotube wall is $30-40 \mathrm{~nm}$ (Figure 4b), and this decreases to $20 \mathrm{~nm}$ in the case of the TiON (Figure 5b). The reason for this significant morphological change is the change in the crystal structure that occurs during the insertion of $\mathrm{N}$ and the removal of $\mathrm{O}$ while $\mathrm{TiO}_{2}$ transforms to TiON. The cross-sectional view of the $\mathrm{TiO}_{2}$ and $\mathrm{TiON}$ nanotubes (Figures $4 c, 5 c)$ additionally shows diameters comparable with measurements in the top-sectional view. Moreover, the higher-magnification images of the top-sectional view of the $\mathrm{TiO}_{2}$ (Figure $4 \mathrm{~d}$ ) and the TiON (Figure 5d) nanotubes reveal small polycrystalline grains with an approximate size of 15-20 nm. The length of the final catalyst in the form of the TiON-Ir nanotubes was determined on crushed particles of the nanotube films that were oriented perpendicular to the 
electron beam of the SEM (not shown). The average measured length is between 1.2 and 1.4 $\mu \mathrm{m}$.
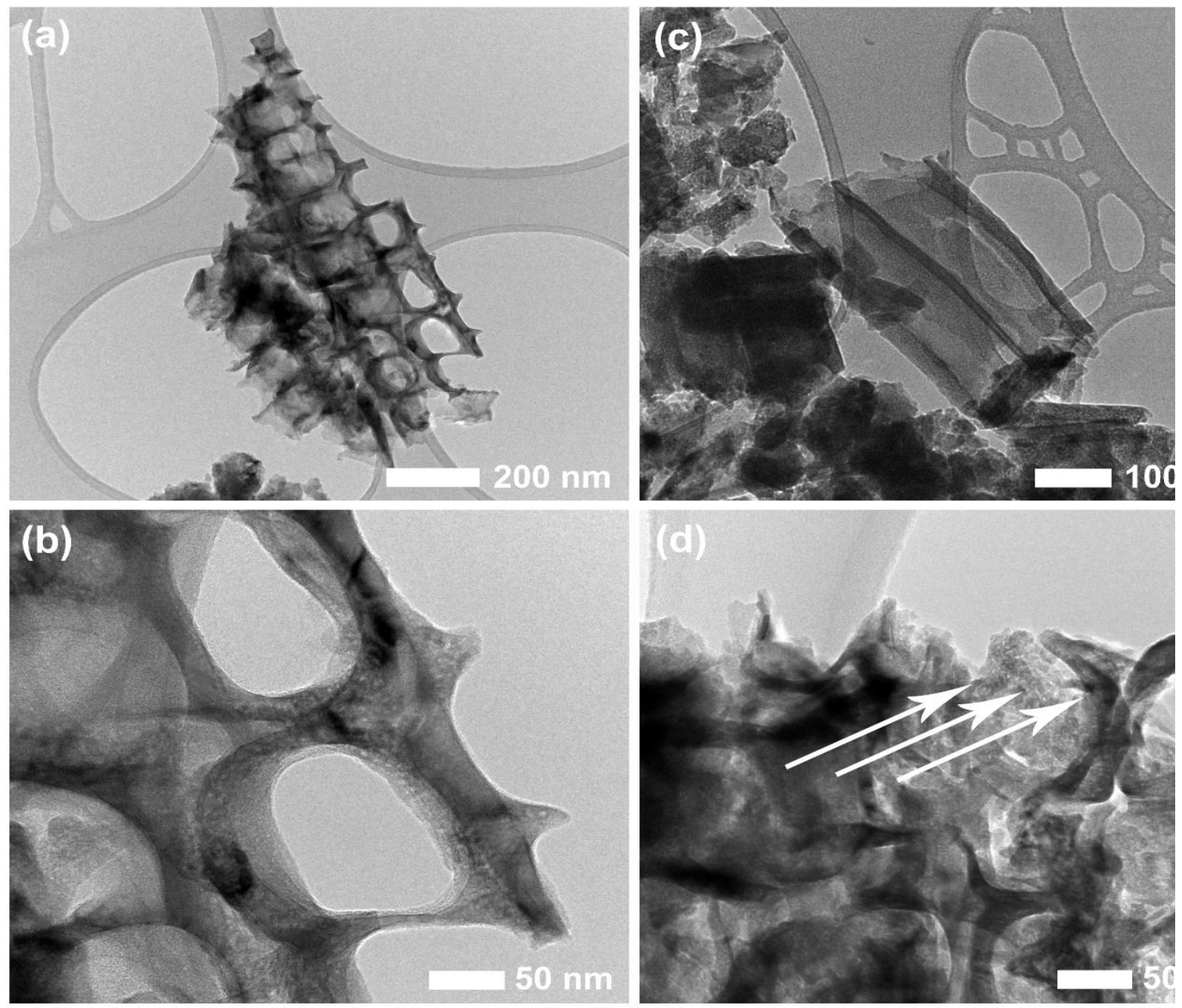

Figure 4: TEM micrographs of $\mathrm{TiO}_{2}$ nanotubes: (a) top-sectional view, (b) higher-magnification top-sectional view, (c) cross-sectional view and (d) high-magnification top-sectional view. 

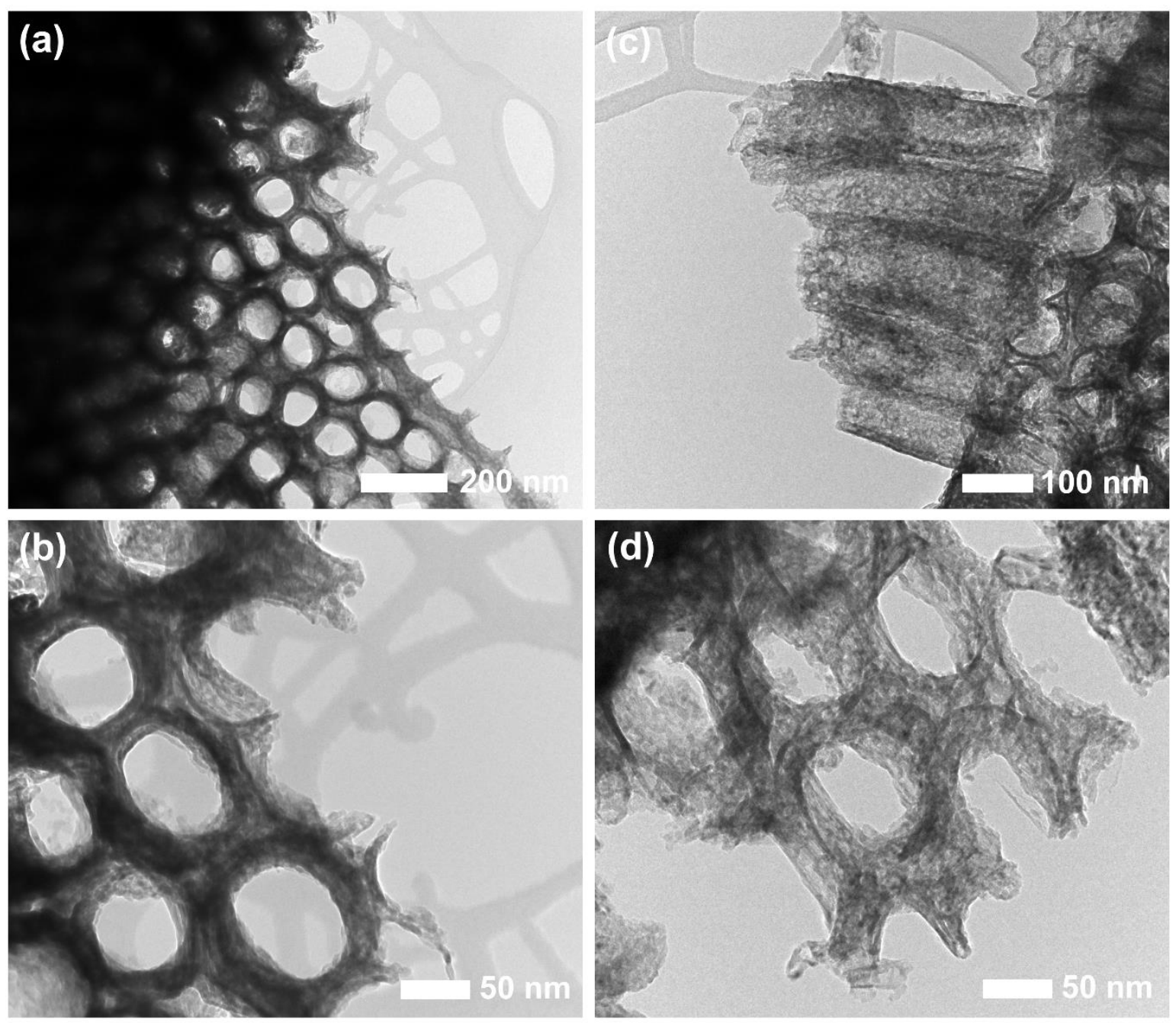

Figure 5: TEM micrographs of TiON nanotubes: (a) top-sectional view, (b) higher-magnification top-sectional view, (c) cross-sectional view and (d) high-magnification top-sectional view.

XRD analyses performed on the same SEM pin stub at all the different synthesis stages for the catalyst preparation show the evolution of the crystallographic structure of the sample. All five diffractograms in Figure 2 show distinct peaks $(\circ)$ related to the hexagonal aluminum titanium vanadium alloy at $2 \theta=35.3^{\circ}(100), 38.5^{\circ}(002), 40.4^{\circ}(101), 53.2^{\circ}(102)$ and $70.9^{\circ}(103)$ angles (PDF 04-002-8708). ${ }^{29}$ The diffraction peaks corresponding to the titanium alloy support (Figure 2a) are observed in each synthesis step due to the small thickness of the nanotubular film. Anodization of the alloy results in uniform $\mathrm{TiO}_{2}$ nanotube arrays with the presence of a small amount of vanadium and aluminum oxides, as shown by the EDS analyses in Figure 6 and by the XPS results (Table 1). $\mathrm{The}^{\mathrm{TiO}_{2}}$ nanotube film grown by anodic oxidation is amorphous, and only the diffraction peaks corresponding to the metal substrate can be observed (Figure $2 b$ ). Annealing at $450^{\circ} \mathrm{C}$ for $1 \mathrm{~h}$ transforms the amorphous nanotubes into the anatase phase (Figure 2c). At the same time, it greatly improves the contact between the titanium alloy and the nanotubular film. ${ }^{40}$ The additional diffraction peaks $(\diamond)$ are related to the anatase phase of the 
tetragonal titanium oxide at $2 \theta=25.4^{\circ}(101), 38.1^{\circ}(004)$ and $54.3^{\circ}(105)$ angles (PDF 01-0706826). ${ }^{29}$ After the annealing in ammonia at $700{ }^{\circ} \mathrm{C}$ the crystalline anatase $\mathrm{TiO}_{2}$ is transformed to TiON (Figure 2d), which is firmly bound to the surface of the alloy due to the preliminary crystallization of the film at $450{ }^{\circ} \mathrm{C}$. However, the surface of the titanium alloy lying beneath the TiON nanotubes is also nitrided to $\mathrm{Ti}_{2} \mathrm{~N}$, as shown in Figure 2d. These additional diffraction peaks are related to $(\bullet)$ tetragonal titanium nitride at $2 \theta=36.3^{\circ}(200)$ and $39.3^{\circ}(111)$ angles (PDF 04-001-9127) ${ }^{29}$ and $(\diamond)$ cubic titanium oxide nitride at $43.1^{\circ}$ (200) angle (PDF 01-0844872). ${ }^{29}$ The XRD spectrum of the sample with Ir nanoparticles is shown in Figure 2e. There is no diffraction peak of the Ir due to the small size and quantity of the Ir nanoparticles. However, their presence was confirmed with the EDS, the electrochemical analyses, and the XPS (Figure $6 b)$. The last of these was used to determine the surface concentrations of the titanium, aluminum, vanadium, oxygen, nitrogen and iridium and their oxidation states before and after the electrochemical measurements. The XPS spectra of the as-prepared sample and the same sample after being used for the electrochemical measurements. The XPS spectra of Ir $4 \mathrm{f}$ and Ti $2 p$ from the as-prepared sample and the same sample after being used for the electrochemical measurements are shown in Figure 6. It is clear that the Ir is mainly present in the metal state (80\%), as evidenced by the $\operatorname{Ir} 4 \mathrm{f}$ peak at $60.8 \mathrm{eV}$, and partially in the $\operatorname{Ir}(4+)$ state (peak at 62.0 $\mathrm{eV}$ ) in the as-prepared sample. ${ }^{41} \mathrm{On}$ this sample the titanium is present in the $\mathrm{Ti}^{4+}$ (46\%, peak at $458.3 \mathrm{eV}), \mathrm{Ti}^{3+}(23 \%$, peak at $457.0 \mathrm{eV})$ and $\mathrm{Ti}^{2+}(31 \%$, peak at $455.6 \mathrm{eV})$ oxidation states related to the $\mathrm{TiO}_{2}$, Ti-O-N and TiN species, respectively. ${ }^{42}$ The nitrogen $\mathrm{N} 1$ s spectrum reveals its presence as nitride and oxynitride, having peaks at 396.5 and $398.0 \mathrm{eV}$. Aluminum and vanadium are in the form of oxides. After the electrochemical test, the surface composition and structure of the same sample are changed significantly. The surface of the TiON substrate is oxidized to a $\mathrm{TiO}_{2}$-like structure $(76 \%)$, but some of the $\mathrm{Ti}-\mathrm{N}-\mathrm{O}(16 \%)$ and $\mathrm{TiN}(8 \%)$ species are still present. The amount of oxygen is increased and the nitrogen is decreased. This finding is important since it proves that the surface $\mathrm{TiON}$ predominantly evolves to $\mathrm{TiO}_{2}$ during the electrochemical operation. Most importantly, the concentration of Ir nanoparticles remains constant, although the Ir's oxidation state is changed to $4+$, as evidenced by the peak at 62.0 $\mathrm{eV}$. Unfortunately, from the XPS spectrum of the Ir $4 \mathrm{f}$ it was not possible to distinguish between the possible presence of the $\mathrm{Ir}^{3+}$ and $\mathrm{Ir}^{4+}$ oxidation states due to the overlapping of these two components, hence we cannot exclude the partial presence of $\mathrm{Ir}^{3+}$ in the sample after the electrochemical test. 
Table 1. Surface composition in at.\% of the sample before and after the electrochemical test using the XPS method

\begin{tabular}{|c|c|c|c|c|c|c|}
\hline Sample & $\mathbf{N}$ & $\mathbf{O}$ & $\mathbf{A l}$ & $\mathbf{T i}$ & $\mathbf{V}$ & $\mathbf{I r}$ \\
\hline Before electrochemical test & 19.8 & 49.5 & 5.2 & 19.7 & 1.9 & 3.8 \\
\hline After electrochemical test & 6.0 & 67.1 & 9.1 & 13.7 & 0.1 & 4.1 \\
\hline
\end{tabular}

(a)

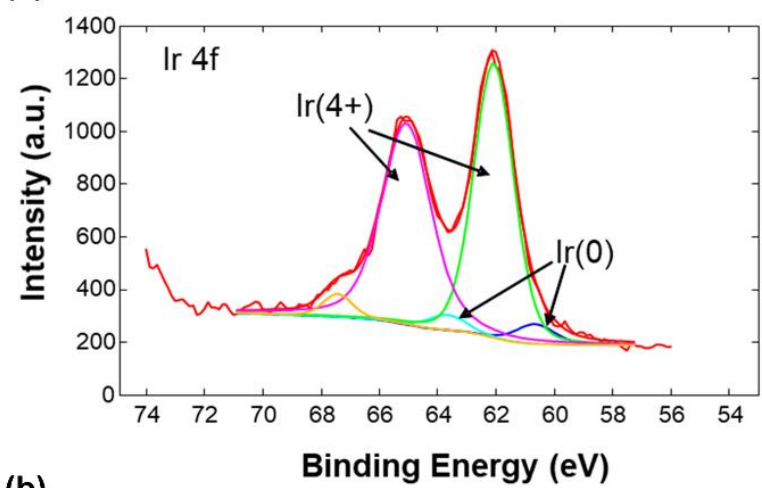

(b)

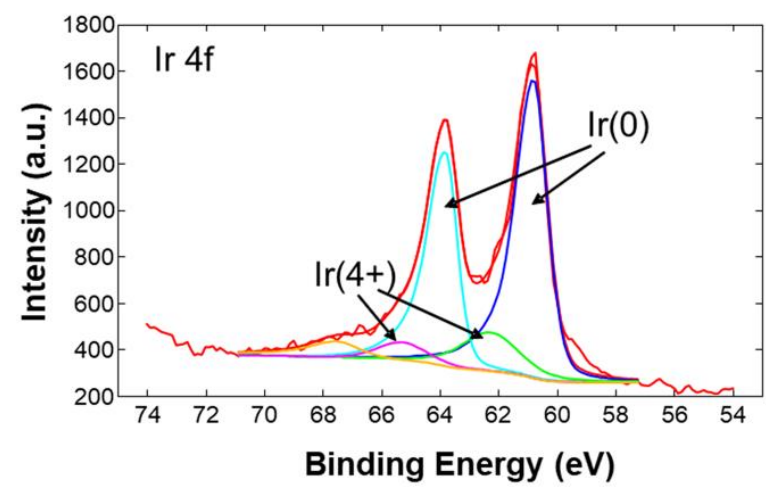

(c)

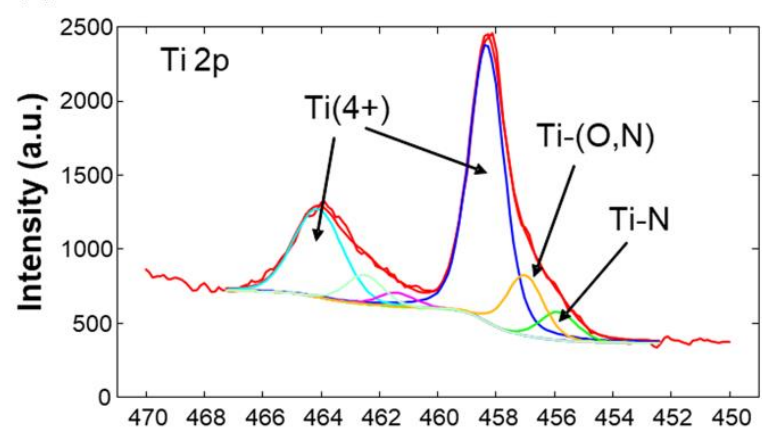

Binding Energy (eV)

(d)

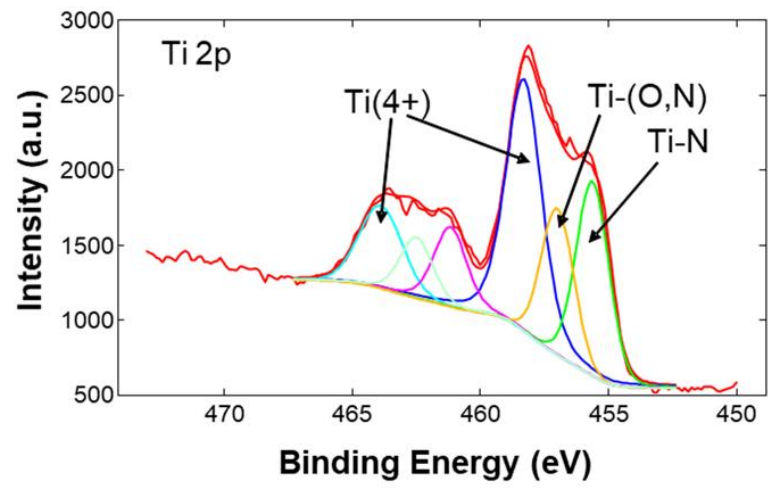

Figure 6. XPS spectra of Ir $4 f$ and Ti $2 p$ acquired on the sample before $(b, d)$ and after the electrochemical test $(a$, c).

The quantity and size of the Ir nanoparticles were determined in the TEM (Figure 7). The average Ir particle size is $2.29 \mathrm{~nm}$. The energy-dispersive X-ray spectroscopy (EDS) of parts with and without the Ir nanoparticles shows their distribution and quantity, which was determined to be 3 at.\% (Figure 7b). This is also in line with the XPS analysis (Table 1). 

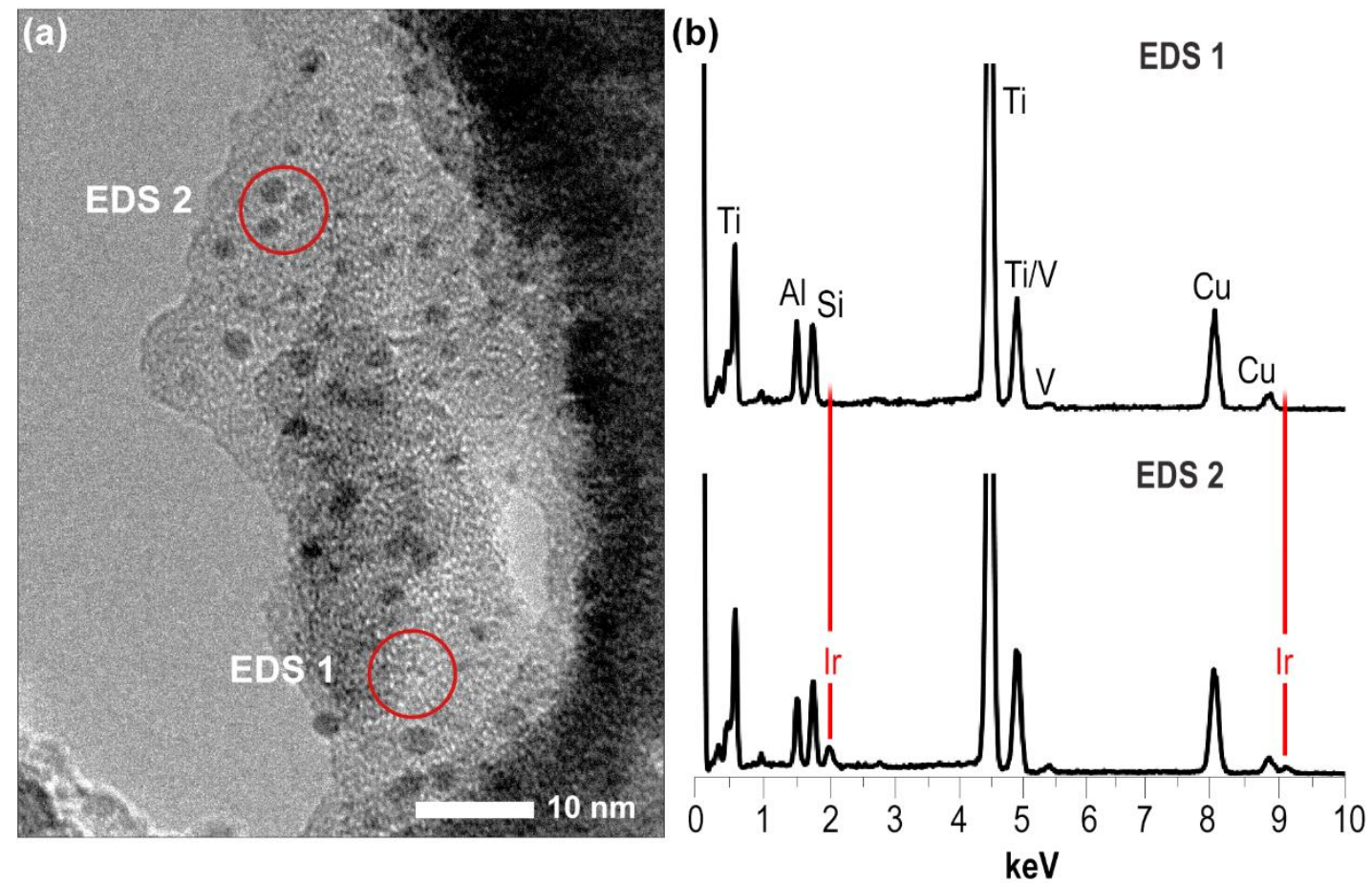

Figure 7: TEM micrograph of TiON nanotube with deposited Ir nanoparticles and corresponding EDS analyses of a part with no Ir nanoparticles (EDS 1) and a part with Ir nanoparticles (EDS 2).

\subsection{DFT calculations of the adhesion of the Ir nanoparticles on the TiON surface}

The adhesion of the Ir nanoparticles on the TiON surface and how the TiON affects their electronic structure were investigated by DFT calculations. To this end, Ir nanoparticles were modeled by small $\operatorname{Ir}_{n}$ clusters $(n=1,4,6,7,12,13,16,19$, and 28) consisting of one to three (111) layers. In addition, we also utilized models of two-layer rods, periodic in one direction, and commensurate overlayers. Snapshots of all these models are shown in Figure S3. Single, standalone Ir atoms adhere most strongly to the TiON surface, with a magnitude of about $5 \mathrm{eV}$, while for larger clusters the magnitude of the adhesion energy decreases as the cluster size increases and reaches about $1 \mathrm{eV} /$ interface-Ir-atom for large clusters (Figure S4a). This reduction is a simple consequence of the fact that the stability of the clusters increases with the cluster size (Figure S4b). A standalone Ir atom can either adsorb into a vacancy of the TiON (note that the crystal structure of TiON contains $25 \%$ of $\mathrm{Ti}$ vacancies) or above the surface, such that it bonds simultaneously with one $\mathrm{O}$ and one $\mathrm{N}$ ion (the two possibilities are very similar in energy, within $0.1 \mathrm{eV}$ ). As for larger Ir ad-clusters, viable adhesion structures are commensurate with the fcc stacking of the TiON support. 
To illustrate the adhesion bonding of the Ir nanoparticles to the $\operatorname{TiON}$, the $\operatorname{Ir}_{[12,10,6]}(111)$ adcluster is analyzed in Figure 8; $\operatorname{Ir}_{[12,10,6]}(111)$ stands for a three-layer cluster consisting of (111) layers with 12, 10, and 6 atoms in the bottom, middle, and top layers, respectively (Figure 8a). The optimized structure of the $\operatorname{Ir}_{[12,10,6]}(111)$ on the TiON is shown in Figure $8 \mathrm{~b}$, while the adhesion bonding is illustrated in Figure $8 \mathrm{c}$ by means of the 3D electron-charge-density difference, $\Delta \rho(\boldsymbol{r})$ of Eq. (1). Note the large charge accumulation (red isosurfaces) around the $\mathrm{N}$ and $\mathrm{O}$ ions below the Ir ad-cluster. A charge depletion of the bottom Ir layer is also evidenced by the blue isosurfaces.
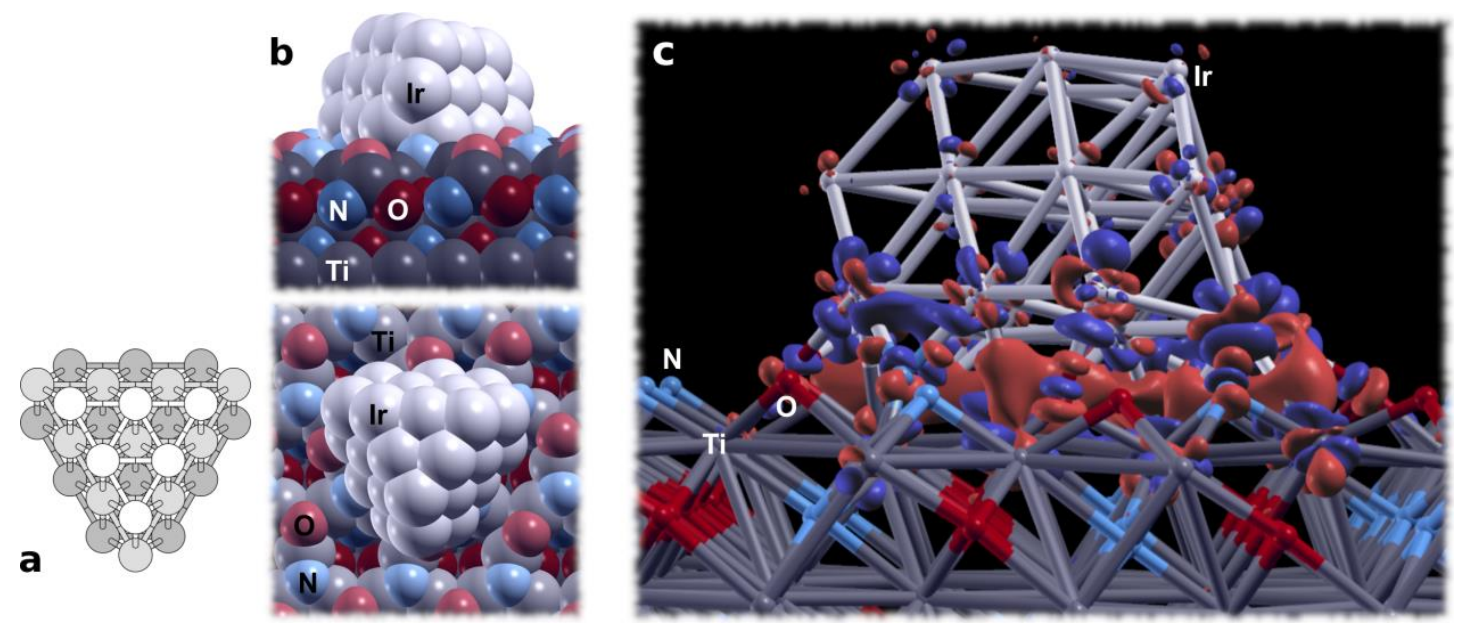

Figure 8: (a) Ideal structure of $\operatorname{Ir}_{[12,10,6]}(111)$ cluster and (b) its optimized structure on TiON. (c) 3D electron-chargedensity difference, $\Delta \rho(\boldsymbol{r})$, of $\operatorname{Ir}[12,10,6](111) / T i O N$, calculated with Eq. (1). Isosurfaces of $\pm 0.01 \mathrm{e} / \mathrm{Bohr}^{3}$ are plotted. The red and blue colors represent the electron-excess and electron-deficit regions, respectively. Note the large electron-charge accumulation at the top $\mathrm{O} / \mathrm{N}$ layer (red isosurfaces) and electron depletion of the bottom Ir layer (blue isosurfaces).

To further analyze the electron-charge transfer due to adhesion of the Ir ad-cluster on TiON, Figure 9 shows the planar integrated electron-charge-density difference along the surface normal direction, $\Delta \rho(z)$ of Eq. (2), superposed with the respective structure so as to facilitate interpretation (blue curve). It is evident that the top Ti layer and the bottom Ir layer loose electron charge $(\Delta \rho(z)<0)$, whereas the top $\mathrm{O} / \mathrm{N}$ layer (red/blue balls) in between the top $\mathrm{Ti}$ and the bottom Ir layers gains electron charge $(\Delta \rho(z)>0)$. The second Ir layer also gains a little electron charge, whereas the third (top) Ir layer is only marginally affected. This is a general pattern of electron-charge redistribution, also observed for other considered ad-clusters not shown here. So as to illuminate the role of the surface $\mathrm{N}$ ions in the adhesion of the Ir nanoparticles on the TiON, the red curve in Figure 9 represents the case where the $\mathrm{N}$ ions below the $\operatorname{Ir}_{[12,10,6]}$ ad-cluster were replaced by $\mathrm{O}$ ions in a stoichiometric 2:3 ratio, i.e., $4 \mathrm{~N}$ were replaced with $6 \mathrm{O}$ and the structure was relaxed. Note that the charge transfer is smaller in this 
case. In particular, the bottom Ir layer donates fewer electrons to the top $\mathrm{O} / \mathrm{N}$ layer. The integration of $\Delta \rho(z)$ in the respective regions reveals that for the blue curve the bottom Ir and top Ti layers loose 1.15 and 0.75 electrons, respectively, whereas the top $\mathrm{O} / \mathrm{N}$ layer gains 1.7 electrons and the middle Ir layer gains 0.2 electrons. For the red curve, which represents the case where $\mathrm{N}$ ions below the Ir cluster are replaced by $\mathrm{O}$ ions, the charge transfer is smaller: the top $\mathrm{O} / \mathrm{N}$ gains 1.25 electrons and the bottom Ir layer looses 0.65 electrons (the respective numbers for the blue curve are 1.7 and 1.15). This clearly suggests that the $\mathrm{N}$ ions at the surface are better docking sites for the Ir nanoparticles than the $\mathrm{O}$ ions. This inference is corroborated by the observation that $\mathrm{N}$ ions also enhance the adhesion strength of the Ir clusters to the surface, compared to the $\mathrm{O}$ ions. In particular, the adhesion energy of the $\operatorname{Ir}_{[12,10,6]}(111)$ cluster to TiON is $-13.6 \mathrm{eV}$ (per cluster), whereas if the $4 \mathrm{~N}$ ions below the Ir cluster are replaced by the $6 \mathrm{O}$ ions, then the adhesion energy is considerably weaker, i.e., $-7.9 \mathrm{eV}$. A similar $\mathrm{N}$ vs. O trend in the charge transfer and the adhesion energy is also observed for the smaller $\operatorname{Ir}_{[7,6,3]}(111)$ cluster (not shown).

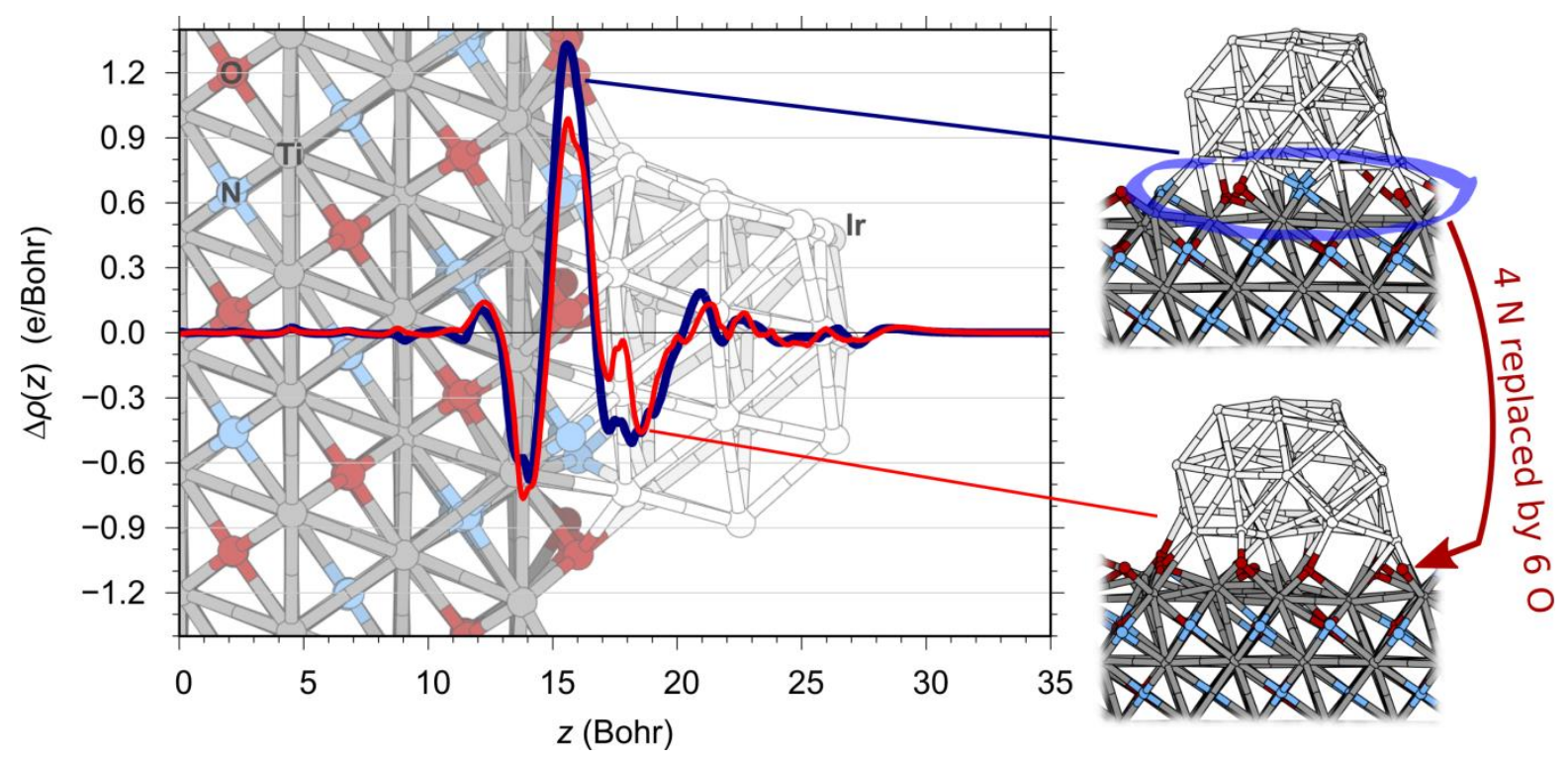

Figure 9: Planar integrated electron-charge-density difference, $\Delta \rho(z)$, for $\operatorname{Ir}_{[12,10,6]}(111)$ on TiON (blue curve), calculated with Eq. (2); $\Delta \rho(z)>0$ means electron accumulation. The respective structure is superposed with the $\Delta \rho(z)$ curve to facilitate interpretation; $z=0$ is arbitrarily set at the fourth Ti layer below the surface. Note that the top Ti layer (dark gray balls) and bottom Ir layer (white balls) loose electron charge $(\Delta \rho(z)<0)$, whereas the top $\mathrm{O} / \mathrm{N}$ layer (red/blue balls) gains electron charge $(\Delta \rho(z)>0)$. The red curve represents the case where the $\mathrm{N}$ ions below the Ir cluster were replaced by $\mathrm{O}$ ions in a stoichiometric 2:3 ratio (i.e., $4 \mathrm{~N}$ were replaced with $6 \mathrm{O}$, as indicated by the sideview snapshots on the right). Note that the charge transfer is smaller in this case. In particular, the bottom Ir layer donates fewer electrons to the top O/N layer.

The bottom line of these arguments is that the adhesion of Ir nanoparticles is enhanced by the $\mathrm{N}$ ions. Furthermore, it is usually assumed that the diffusion energy of the nanoparticles on the 
surface scales with the adhesion energy, i.e., the stronger is the adhesion, the larger is the diffusion barrier. This implies that the presence of $\mathrm{N}$ ions reduces the tendency of the $\mathrm{Ir}$ nanoparticles to sinter. Having small nanoparticles appears to be important, not only because in this way the fraction of surface Ir atoms is larger, but because the analysis presented above reveals that the TiON substrate affects predominantly the electronic structure of the bottommost Ir layer, the layer above it is affected to a much smaller extent, whereas the modification of the third layer is already vanishing. Note that for more than two layers thick nanoparticles, only the "perimeter" sites of the two bottommost layers are exposed to the exterior environment. Provided that these perimeter sites are catalytically superior, this suggests that the nanoparticles should be small or the fraction of such perimeter sites is negligible.

\subsection{Electrochemical performance}

In this work the OER polarization curves are normalized per unit mass of iridium. This is due to several issues related to determining the electrochemically active surface area (ECSA) of the supported Ir-based electrocatalyst, which are further explained in the Supporting Information (see Section S4). A rapid and accurate evaluation of the ECSA remains a challenging task. After many attempts in the literature with limited success, only recently Watzele et al. demonstrated an estimation of the real electroactive surface area of supported, nanostructured, oxide electrocatalysts using impedance measurements. ${ }^{43}$ This concept is based on acquiring the values of the adsorption capacitance. We note that in our case the loading of Ir on high-surface-area TiON is extremely low $\left(1.7 \mu \mathrm{g}_{\mathrm{Ir}} \mathrm{cm}^{-2}\right.$ geom $)$; hence the relative portion of the TiON surface area is large. This makes obtaining a reliable value of the adsorption capacitance for the TiON-Ir composite highly challenging. Therefore, a proper methodology for the composites, such as the one roughly proposed here, is yet to be developed and is a matter of ongoing investigation. This is one of the reasons that in this study we present the data as "current per mass of iridium". The other reason for such a presentation is that the mass activities are the most relevant parameter for real-application purposes. Nevertheless, a rough ECSA estimation was provided with the TEM analysis $\left(\sim 87 \mathrm{~m}^{2} \mathrm{~g}_{\mathrm{Ir}}{ }^{-1}\right.$; for details of determination see Supporting Information, Section S5). Such values are relatively similar to the ECSA of supported Ir nanoparticles. ${ }^{30,43}$ In order to place the catalytic performance of the TiON-Ir composite in the context of state-of-the-art OER electrocatalysts, its performance was compared to a commercial, non-supported, Ir analogue (Ir Black) with the ECSA ranging between $\sim 23$ and $30 \mathrm{~m}^{2} \mathrm{~g}_{\mathrm{Ir}}{ }^{-1} \cdot{ }^{30,44}$ The OER performance of the Ir Black analogue was evaluated under a rotating-disc-electrode (RDE) configuration (for details see the experimental section). 
The OER performances are compared in the kinetic region (up to $1.6 \mathrm{~V}$, Figure 10a), because at higher potentials the OER polarization curve is not solely governed by its kinetics, but transport and the removal of the oxygen bubbles from the surface influence the current response. The comparison reveals that the TiON-Ir analogue significantly outperforms the Ir Black catalyst (Figure 10a solid line). In order to explain the observed activity trends, several characteristics should be considered. Firstly, the utilization of the iridium's ECSA is considerably better in the case of TiON-Ir (this is in line with approximately 4-fold-larger ECSA, see above), which results in a higher mass activity. Secondly, in comparison to TiONIr, the Ir Black analogue has a lower electron mobility, since the electrode is prepared from a film of nanoparticles and does not allow one-dimensional charge transport, as in the case of vertically oriented nanotube-based films. ${ }^{45} \mathrm{~A}$ similar effect has also been noticed in the case of TiON-Ir powder in our previous study. ${ }^{46}$ On the other hand, it is important to stress that the average particle size of both analogues is very similar, i.e., $2.29 \mathrm{~nm}$ for TiON-Ir and $2.31 \mathrm{~nm}$ for Ir Black, see Supporting Information, Sections S5 and S6). Hence, any particle-sizedependent activity effects should be ruled out when comparing the activities of both analogues. Last but not least, DFT calculations, presented in the previous section, confirm the existence of a strong interaction (commonly known as SMSI) between the Ir and the TiON support. The benefits of SMSI in terms of catalytic activity were shown previously for other systems $\mathbf{s}^{47,48,49}$ and are now being progressively recognized also in the particular case of OER kinetics. ${ }^{6,50}$

To further elucidate the activity difference, a Tafel slope analysis was performed (Figure 10b). Interestingly, the Tafel slope for TiON-Ir $(76 \mathrm{mV} / \mathrm{dec})$ is somewhat higher than expected ( $40-50 \mathrm{mV} / \mathrm{dec}$ is common for Ir oxides ${ }^{30}$ ). By comparison, in the case of the Ir Black analogue a slope of $54 \mathrm{mV}$ per decade was obtained (Figure 10b). As mentioned, this difference cannot be due to the particle size, as it is almost identical for both samples. The same is true for other influential surface-related parameters such as facets, kinks, edges, and steps. Based on the present DFT calculations and also referring to the literature data on similar systems, ${ }^{49,50,6}$ it is reasonable to suggest that the OER mechanism, and thus the Tafel slope, are changed due to the SMSI-like interaction between the Ir nanoparticles and the support. It has been shown previously that an unusually large Tafel slope can be associated with the SMSI effect when using semiconducting catalytic materials such as $\mathrm{TiO}_{2}$ (see also Table $\mathrm{S} 1$ ). ${ }^{51}$ In this work, we also demonstrated a similar SMSI effect for the TiON-Ir system, so the steep Tafel slope could potentially be ascribed to this phenomenon. On the other hand, we should not rule out the possibility that at least partly the steep Tafel slope is due to an electrochemically formed surface layer of $\mathrm{TiO}_{2}$ (as indicated by XPS analysis, see Table 1). ${ }^{51}$ 
Additionally, we assume that at least to some extent the high performance of the present IrTiON system can be ascribed to the different composition of the Ir valence state in comparison to the Ir Black analogue, i.e., the presence of a larger number of Ir species with a mixed valence $\left(\operatorname{Ir}^{3+/ 5+}\right)$, which have been shown to be the OER-active surface states of iridium electrodes. ${ }^{52,53,54,55,41,56}$ Although such species could neither be confirmed nor refuted by the present XPS analysis (see Table 1 and the associated text), their presence was indirectly demonstrated in our previous study, which showed that the TiON support significantly affected the Ir redox chemistry and the related transient behavior of the oxide layer by preserving the lower Ir oxidation states. ${ }^{46}$

To induce electrode degradation, a potentiostatic treatment at $1.8 \mathrm{~V}$ vs RHE was employed (we remark again that a degradation time of $1 \mathrm{~h}$ for the TiON-Ir and $30 \mathrm{~min}$ for the Ir-Black was used). Note that this potential is significantly higher than normally used when simulating the practical working conditions of the catalyst layer. Here, we used high potentials in order to evaluate the durability in a relatively rapid manner. Obviously, the TiON-Ir analogue significantly outperforms the Ir Black catalyst (Figure 10a, dashed lines). This is in good agreement with the present DFT calculations, which reveal that the adhesion of Ir nanoparticle is enhanced by the presence of $\mathrm{N}$ ions. The stronger the adhesion, the larger is the diffusion barrier, and thus the better the general stability of the material as particle coalescence is diminished. In fact, rather surprisingly, in the case of TiON-Ir, the OER activity even slightly increased after the potentiostatic degradation, when compared to the initial activity. Generally, we would expect at least some performance loss, especially at potentials of such magnitude (1.8 $\mathrm{V}$ vs RHE), which typically trigger the formation of higher-valent Ir oxide layers that compromise the catalyst's stability. We ascribe the slightly increased performance to the generation of highly active, porous, hydrated oxide ${ }^{57,55}$ during an anodic perturbation. Note that the degree of hydration correlates with the OER activity due to the enhanced formation of vicinal $\mathrm{Ir}^{5+}$ sites. ${ }^{57,55} \mathrm{In}$ any case, such a performance trend is fascinating and indirectly proves that hydrated porous Ir can sustain the lowering of the Ir's oxidation state, and hence survive a very harsh electrochemical treatment, if it is in contact with an appropriate stable support (either TiON or a thin layer of $\mathrm{TiO}_{2}$ ). Of course, more dedicated studies are needed to elucidate this unexpected effect.

Morphological changes were inspected with the IL-SEM imaging. No significant changes to the TiON support during the degradation protocol were observed. We note that the particle size of the Ir was below the SEM resolution, and hence no changes before and after the electrocatalytic measurements could be observed (Figure S8). 
In summary, the superior activity is ascribed to the well-dispersed Ir nanoparticles on the TiON support that stem from the strong anchoring of the Ir on N-sites, evidenced by DFT calculations. An additional contributing effect is the SMSI-like interaction, which induces more Ir species with mixed valence. The unusually steep Tafel slope can be ascribed either to the nature of the TiON itself or alternatively to the presence of a semiconducting $\mathrm{TiO}_{2}$ surface layer that was detected with the XPS analysis. After the degradation protocol no loss of OER activity was observed. This means that no coalescence of Ir particles occurs, which is in excellent agreement with the results of the DFT analysis. In fact, the activity after the "degradation" was even higher, which can be explained by an increased degree of hydration. Again, this observation can be ascribed to the SMSI effect, which prevents the oxidative degradation of Ir particles.

To the best of our knowledge, the present mass activity of TiON-Ir is the highest reported so far for OER catalysts consisting of pure Ir on a support (See Table S1). In fact, the only Ir-based catalysts with higher activity are the recently reported bimetallic Ir-Ni and Ir-Co nanowires ${ }^{58,59}$ and Ir networks, ${ }^{60}$ all of which belong to more complex materials, so a direct comparison is not possible. It is also important to note that in the present investigation a much more aggressive electrochemical treatment ( $1.8 \mathrm{~V}$ vs RHE) was used for the stability testing in comparison to the above-mentioned reports. Finally, we should note that in the case of the TiON-Ir the Ir geometric loading is approximately 150-fold smaller than in PEM electrolysers with the best state-of-the-art performance. ${ }^{10}$

(a)

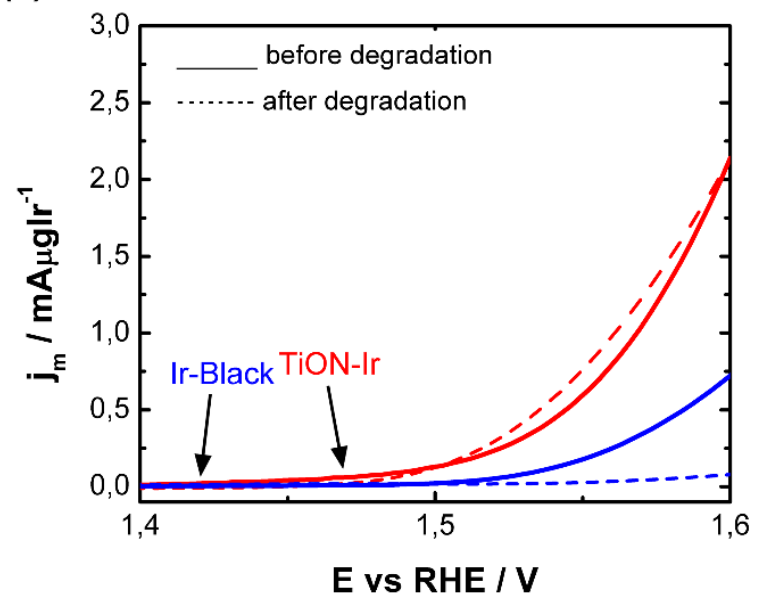

(b)

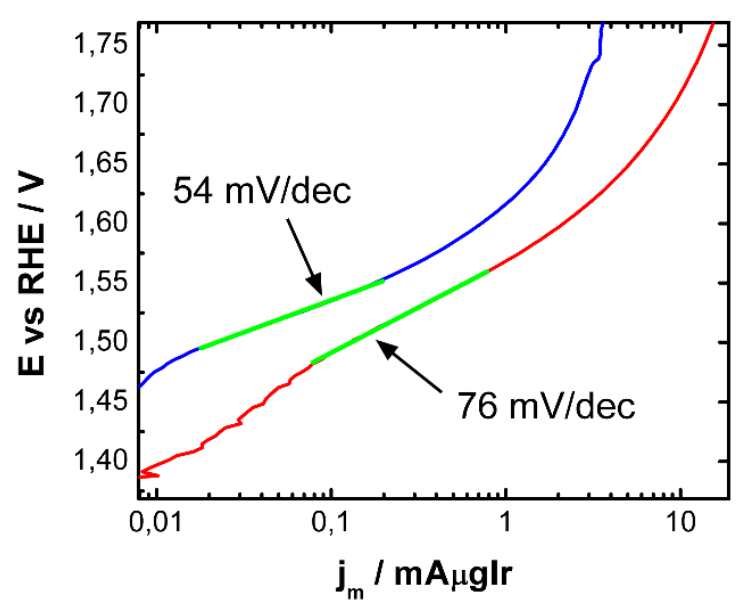

Figure 10. OER polarization curves of TiON-Ir (black line) and Ir-Black (grey line) analogues before (solid line) and after (dash line) degradation protocol. (a) LSV response $\left(20 \mathrm{mVs}^{-1}\right)$ and (b) Tafel analysis. 


\section{Conclusions}

The present investigation introduces a novel approach to the preparation and characterization of thin-film electrode composites. As a case example, a novel oxygen-evolution composite consisting of a thin-film electrode based on iridium nanoparticles dispersed on a nanotubular, high-surface-area, oxynitride support (TiON-Ir) is presented. The approach made possible an elegant characterization using the identical-location scanning electron microscopy (IL-SEM) and X-ray diffraction (XRD) techniques. In this way, the morphological and compositional changes to the TiON-Ir were successfully tracked through the whole synthesis and electrocatalytic processes. The mass activity of the present TiON-Ir material for OER is one of the best measured so far for this reaction. Furthermore, employing a rigorous degradation protocol, a remarkable electrochemical stability of the TiON-Ir composite was demonstrated as well. Importantly, the TiON support itself did not suffer any morphological changes during the electrochemical treatment. Based on complementary DFT modelling, the enhanced electrochemical performance is ascribed to a strong metal-support interaction (SMSI) between the Ir and the TiON. The presence of protective oxides, which hinders excessive oxidation of Ir, is an additional factor that can explain the excellent performance.

\section{Supporting Information}

The Supporting Information is available free of charge on the RSC Publication website at DOI: A comparison of the electrochemical activity of the TiON-Ir and $\mathrm{TiO}_{2}$-Ir, SEM of the TiON-Ir catalyst before and after the electrocatalytic measurements, morphological changes throughout the synthesis process in a video format, TEM analysis for particle size determination, additional results of DFT calculations, comparison of characteristic OER parameters with the state of the art.

\section{Author contributions}

The manuscript was written through contributions of all authors. All authors have given approval to the final version of the manuscript. M. B., L. S., N. H. and P. J. conceived the idea and designed the experiments. L. S., Ž. M. and M. B. synthesized catalysts and prepared samples for materials and electrochemical characterization. Electrochemical characterization was performed by P. J. SEM characterization was performed by M. B. and L. S. X-ray 
photoelectron spectroscopy was performed by J. K. TEM characterization was performed by S. D., G. K. P. and G. D. ICP-OES measurements were performed by V. S. Š. X-ray diffraction data were analyzed by M. B. and Ž. M. DFT calculations were performed by A. K. The project was supervised by N. H., M. G., M. B., L. S. and P. J.

\section{Conflicts of interest}

The authors declare no competing financial interests.

\section{Acknowledgments}

The provision of financial support for the research and the preparation of the manuscript by the Slovenian Research Agency (ARRS) within the research programs P2-0084, P2-0152 and P20393 and projects Z2-8161, N2-0106 and Z1-9165 is gratefully acknowledged. The authors also thank Edi Kranjc (Department of Inorganic Chemistry and Technology, National Institute of Chemistry, Slovenia) for the X-ray powder-diffraction measurements.

\section{Notes and references}

$\$ \quad$ The structural model for the $\mathrm{Ti}_{1.5} \mathrm{ON}$ bulk was constructed based on data for $\operatorname{Ti}_{0.7}\left(\mathrm{~N}_{0.33} \mathrm{O}_{0.67}\right)$ (ICSD 426340), ${ }^{61}$ with Fm-3m structure and unit cell with $a=4.199 \AA$. From the EELS spectra of the investigated samples we measured that the concentrations of oxygen and nitrogen are approximately equal and therefore a small deviation from the original unit cell parameters was expected. Throughout the text we used the term TiON for the approximate formula $\mathrm{Ti}_{1.5} \mathrm{ON}$.

1 J. C. Meier, C. Galeano, I. Katsounaros, J. Witte, H. J. Bongard, A. A. Topalov, C. Baldizzone, S. Mezzavilla, F. Schüth and K. J. J. Mayrhofer, Beilstein J. Nanotechnol., 2014, 5, 44-67.

2 P. Lettenmeier, J. Majchel, L. Wang, V. A. Saveleva, S. Zafeiratos, E. R. Savinova, J.-J. Gallet, F. Bournel, A. S. Gago and K. A. Friedrich, Chem. Sci., 2018, 9, 3570-3579.

3 L. C. Seitz, C. F. Dickens, K. Nishio, Y. Hikita, J. Montoya, A. Doyle, C. Kirk, A. Vojvodic, H. Y. Hwang, J. K. Norskov and T. F. Jaramillo, Science (80-. )., 2016, 353, 1011-1014.

4 L. Wang, F. Song, G. Ozouf, D. Geiger, T. Morawietz, M. Handl, P. Gazdzicki, C. Beauger, U. Kaiser, R. Hiesgen, A. S. Gago and K. A. Friedrich, J. Mater. Chem. A, 2017, 5, 3172-3178.

5 H. S. Oh, H. N. Nong and P. Strasser, Adv. Funct. Mater., 2015, 25, 1074-1081.

6 H.-S. Oh, H. N. Nong, T. Reier, A. Bergmann, M. Gliech, J. Ferreira de Araújo, E. Willinger, R. Schlögl, D. Teschner and P. Strasser, J. Am. Chem. Soc., 2016, 138, 12552-12563.

7 H. N. Nong, H.-S. Oh, T. Reier, E. Willinger, M.-G. Willinger, V. Petkov, D. Teschner and P. 
Strasser, Angew. Chem. Int. Ed. Engl., 2015, 54, 2975-9.

8 S. Trasatti, Electrochim. Acta, 2000, 45, 2377-2385.

9 T. Audichon, T. W. Napporn, C. Canaff, C. Morais, C. Comminges and K. B. Kokoh, J. Phys. Chem. C, 2016, 120, 2562-2573.

10 K. A. Lewinski, D. van der Vliet and S. M. Luopa, ECS Trans., 2015, 69, 893-917.

11 P. C. K. Vesborg and T. F. Jaramillo, RSC Adv., 2012, $2,7933$.

12 L. Castanheira, L. Dubau, M. Mermoux, G. Berthome, N. Caque, E. Rossinot and M. Chatenet, ACS Catal., 2014, 4, 2258-2267.

13 L. Castanheira, W. O. Silva, F. H. B. Lima, A. Crisci, L. Dubau and F. Maillard, ACS Catal., 2015, 5, 2184-2194.

14 H. S. Oh, H. N. Nong, T. Reier, A. Bergmann, M. Gliech, J. Ferreira De Araújo, E. Willinger, R. Schlögl, D. Teschner and P. Strasser, J. Am. Chem. Soc., 2016, 138, 12552-12563.

15 M. Ledendecker, S. Geiger, K. Hengge, J. Lim, S. Cherevko, A. M. Mingers, D. Göhl, G. V. Fortunato, D. Jalalpoor, F. Schüth, C. Scheu and K. J. J. Mayrhofer, Nano Res., 2019, 12, 2275-2280.

16 Y. R. Smith, R. S. Ray, K. Carlson, B. Sarma and M. Misra, Mater. (Basel, Switzerland), 2013, 6, 2892-2957.

17 S. DeWitt and K. Thornton, 2015, pp. 19-39.

18 P. Roy, S. Berger and P. Schmuki, Angew. Chemie Int. Ed., 2011, 50, 2904-2939.

19 M. K. Nowotny, T. Bak and J. Nowotny, J. Phys. Chem. B, 2006, 110, 16270-16282.

20 N. P. Padture and X. Wei, J. Am. Ceram. Soc., 2004, 86, 2215-2217.

21 J. Zhang, J. H. Bang, C. Tang and P. V Kamat, ACS Nano, 2010, 4, 387-395.

22 K. Z. Soderznik, C. Fabrega, F. Hernandez-Ramirez, J. D. Prades, M. Čeh, K. Z. Soderznik, C. Fabrega, F. Hernandez-Ramirez, J. D. Prades and M. Čeh, Proceedings, 2019, 15, 9.

23 M. Chisaka, Phys. Chem. Chem. Phys., 2018, 20, 15613-15617.

24 L. Suhadolnik, D. Lašič Jurković, B. Likozar, M. Bele, S. Drev and M. Čeh, Appl. Catal. B Environ., 2019, 257, 117894.

25 A. Lončar, L. Moriau, K. Stojanovski, F. Ruiz-Zepeda, P. Jovanovic, M. Bele, M. Gaberscek and N. Hodnik, J. Phys. Energy, 2020, 0-27.

26 M. Zorko, B. Jozinović, M. Bele, N. Hodnik and M. Gaberšček, Ultramicroscopy, 2014, 140, $44-50$.

27 N. Hodnik and S. Cherevko, Curr. Opin. Electrochem., 2019, 15, 73-82.

28 N. Hodnik, M. Zorko, M. Bele, S. Hočevar and M. Gaberšček, J. Phys. Chem. C, 2012, 116, 21326-21333.

29 Kabekkodu, 2016.

30 S. M. Alia, B. Rasimick, C. Ngo, K. C. Neyerlin, S. S. Kocha, S. Pylypenko, H. Xu and B. S. Pivovar, J. Electrochem. Soc., 2016, 163, F3105-F3112.

P. Giannozzi, O. Andreussi, T. Brumme, O. Bunau, M. Buongiorno Nardelli, M. Calandra, R. 
Car, C. Cavazzoni, D. Ceresoli, M. Cococcioni, N. Colonna, I. Carnimeo, A. Dal Corso, S. de Gironcoli, P. Delugas, R. A. DiStasio, A. Ferretti, A. Floris, G. Fratesi, G. Fugallo, R. Gebauer, U. Gerstmann, F. Giustino, T. Gorni, J. Jia, M. Kawamura, H.-Y. Ko, A. Kokalj, E.

Küçükbenli, M. Lazzeri, M. Marsili, N. Marzari, F. Mauri, N. L. Nguyen, H.-V. Nguyen, A. Otero-de-la-Roza, L. Paulatto, S. Poncé, D. Rocca, R. Sabatini, B. Santra, M. Schlipf, A. P. Seitsonen, A. Smogunov, I. Timrov, T. Thonhauser, P. Umari, N. Vast, X. Wu and S. Baroni, J. Phys. Condens. Matter, 2017, 29, 465901.

V. I. Anisimov, J. Zaanen and O. K. Andersen, Phys. Rev. B, 1991, 44, 943-954.

M. Cococcioni and S. De Gironcoli, Phys. Rev. B - Condens. Matter Mater. Phys., , DOI:10.1103/PhysRevB.71.035105.

J. P. Perdew, K. Burke and M. Ernzerhof, Phys. Rev. Lett., 1996, 77, 3865-3868.

A. Dal Corso, Comput. Mater. Sci., 2014, 95, 337-350.

pslibrary.

I. Timrov, N. Marzari and M. Cococcioni, Phys. Rev. B, 2018, 98, 085127.

M. Methfessel and A. T. Paxton, Phys. Rev. B, 1989, 40, 3616-3621.

M. Sluban, P. Umek, Z. Jagličić, J. Buh, P. Šmitek, A. Mrzel, C. Bittencourt, P. Guttmann, M.H. Delville, D. Mihailović and D. Arčon, ACS Nano, 2015, 9, 10133-10141.

40 M. Sarraf, A. Razak, R. Crum, C. Gámez, B. Ramirez, N. Kasim, B. Nasiri-Tabrizi, V. Gupta, N. Sukiman and W. Basirun, Process. Appl. Ceram., 2017, 11, 311-321.

V. Pfeifer, T. E. Jones, J. J. Velasco Vélez, C. Massué, R. Arrigo, D. Teschner, F. Girgsdies, M. Scherzer, M. T. Greiner, J. Allan, M. Hashagen, G. Weinberg, S. Piccinin, M. Hävecker, A. Knop-Gericke and R. Schlögl, , DOI:10.1002/sia.5895.

J. H. Han and J. H. Bang, J. Mater. Chem. A, 2014, 2, 10568-10576.

S. Watzele, P. Hauenstein, Y. Liang, S. Xue, J. Fichtner, B. Garlyyev, D. Scieszka, F. Claudel, F. Maillard and A. S. Bandarenka, ACS Catal., , DOI:10.1021/acscatal.9b02006.

S. M. Alia, K. E. Hurst, S. S. Kocha and B. S. Pivovar, J. Electrochem. Soc., 2016, 163, F3051-F3056.

W.-R. Kim, H. Park and W.-Y. Choi, Nanoscale Res. Lett., 2015, 10, 63.

M. Bele, K. Stojanovski, P. Jovanovič, L. Moriau, G. Koderman Podboršek, J. Moškon, P. Umek, M. Sluban, G. Dražič, N. Hodnik and M. Gaberšček, ChemCatChem, 2019, 11, 50385044.

P. Chen, A. Khetan, F. Yang, V. Migunov, P. Weide, S. P. Stürmer, P. Guo, K. Kähler, W. Xia, J. Mayer, H. Pitsch, U. Simon and M. Muhler, ACS Catal., 2017, 7, 1197-1206.

K. Foger and J. R. Anderson, Appl. Catal., 1986, 23, 139-155.

G. Chen, S. R. Bare and T. E. Mallouk, J. Electrochem. Soc., 2002, 149, A1092.

50 T. Reier, D. Teschner, T. Lunkenbein, A. Bergmann, S. Selve, R. Kraehnert, R. Schlögl and P. Strasser, J. Electrochem. Soc., 2014, 161, F876-F882.

51 A. G. Scheuermann, J. D. Prange, M. Gunji, C. E. D. Chidsey and P. C. McIntyre, Energy Environ. Sci., 2013, 6, 2487-2496. 
Chem. Sci., 2014, 5, 3591.

53 V. Pfeifer, T. E. Jones, S. Wrabetz, C. Massué, J. J. Velasco Vélez, R. Arrigo, M. Scherzer, S. Piccinin, M. Hävecker, A. Knop-Gericke and R. Schlögl, Chem. Sci., 2016, 7, 6791-6795.

54 V. Pfeifer, T. E. Jones, J. J. Velasco Vélez, R. Arrigo, S. Piccinin, M. Hävecker, A. KnopGericke and R. Schlögl, Chem. Sci., , DOI:10.1039/C6SC04622C.

55 V. Pfeifer, T. E. Jones, J. J. Velasco Vélez, C. Massué, M. T. Greiner, R. Arrigo, D. Teschner, F. Girgsdies, M. Scherzer, J. Allan, M. Hashagen, G. Weinberg, S. Piccinin, M. Hävecker, A. Knop-Gericke and R. Schlögl, Phys. Chem. Chem. Phys., 2016, 18, 2292-2296.

56 O. Kasian, S. Geiger, T. Li, J. Grote, K. Schweinar, S. Zhang, C. Scheu, D. Raabe, S. Cherevko, B. Gault and K. J. J. Mayrhofer, , DOI:10.1039/c9ee01872g.

57 A. Minguzzi, C. Locatelli, O. Lugaresi, E. Achilli, G. Cappelletti, M. Scavini, M. Coduri, P. Masala, B. Sacchi, A. Vertova, P. Ghigna and S. Rondinini, ACS Catal., 2015, 5, 5104-5115.

58 S. M. Alia, S. Shulda, C. Ngo, S. Pylypenko and B. S. Pivovar, ACS Catal., 2018, 8, $2111-$ 2120 .

59 F. Godínez-Salomón, L. Albiter, S. M. Alia, B. S. Pivovar, L. E. Camacho-Forero, P. B. Balbuena, R. Mendoza-Cruz, M. J. Arellano-Jimenez and C. P. Rhodes, ACS Catal., 2018, 8, 10498-10520.

60 A. W. Jensen, G. W. Sievers, K. D. Jensen, J. Quinson, J. A. Arminio-Ravelo, V. Brüser, M. Arenz and M. Escudero-Escribano, J. Mater. Chem. A, 2020, 8, 1066-1071.

61 S. G. Seo, C. H. Park, H. Y. Kim, W. H. Nam, M. Jeong, Y. N. Choi, Y. S. Lim, W. S. Seo, S. J. Kim, J. Y. Lee and Y. S. Cho, J. Mater. Chem. A, 2013, 1, 3639-3644. 